\title{
Evaluation of de novo transcriptome assemblies from RNA-Seq data
}

\author{
Bo $\mathrm{Li}^{1 \dagger}$, Nathanael Fillmore ${ }^{2 \dagger}$, Yongsheng Bai ${ }^{3}$, Mike Collins ${ }^{4}$, James A Thomson ${ }^{4,5,6}$, Ron Stewart ${ }^{4}$ \\ and Colin N Dewey ${ }^{2,7^{*}}$
}

\begin{abstract}
De novo RNA-Seq assembly facilitates the study of transcriptomes for species without sequenced genomes, but it is challenging to select the most accurate assembly in this context. To address this challenge, we developed a model-based score, RSEM-EVAL, for evaluating assemblies when the ground truth is unknown. We show that RSEM-EVAL correctly reflects assembly accuracy, as measured by REF-EVAL, a refined set of ground-truth-based scores that we also developed. Guided by RSEM-EVAL, we assembled the transcriptome of the regenerating axolotl limb; this assembly compares favorably to a previous assembly. A software package implementing our methods, DETONATE, is freely available at http://deweylab.biostat.wisc.edu/detonate.
\end{abstract}

\section{Background}

RNA sequencing (RNA-Seq) technology is revolutionizing the study of species that have not yet had their genomes sequenced by enabling the large-scale analysis of their transcriptomes. To study such transcriptomes, one must first determine a set of transcript sequences via de novo transcriptome assembly, which is the reconstruction of transcript sequences from RNA-Seq reads without the aid of genome sequence information. A number of de novo transcriptome assemblers are currently available, many designed for Illumina platform data [1-8] and others targeted for Roche 454 Life Science platform data [9-12]. These assemblers, combined with their often sizable sets of user-tunable parameters, enable the generation of a large space of candidate assemblies for a single data set. However, appropriately evaluating the accuracy of assemblies in this space, particularly when the ground truth is unknown, has remained challenging.

A number of studies have been devoted to the evaluation of de novo transcriptome assemblies [13-20]. Assembly evaluation measures used in such studies can be

\footnotetext{
*Correspondence: cdewey@biostat.wisc.edu

${ }^{\dagger}$ Equal contributors

2 Department of Computer Sciences, University of Wisconsin, Madison, WI 53706, USA

${ }^{7}$ Department of Biostatistics and Medical Informatics, University of Wisconsin, Madison, WI 53706, USA

Full list of author information is available at the end of the article
}

grouped into two classes: reference-based and referencefree. Reference-based measures are those that are computed using previously known sequences. For example, after establishing a correspondence between assembly elements and a reference transcript set, one can calculate the fraction of assembly elements that accurately match a reference transcript (precision), the fraction of reference transcripts that are matched by assembly elements (recall), or a combination of these two (e.g., the $F_{1}$ measure) $[5,16,17]$. In addition to transcript sets, genome and protein sequences can also be used as references for assembly evaluation $[2,4,8,13,15,20]$.

However, in most cases where de novo assembly is of interest, reference sequences are either not available, incomplete or considerably diverged from the ground truth of a sample of interest, which makes the assembly evaluation task markedly more difficult. In such cases, one must resort to reference-free measures. Commonly used reference-free measures include median contig length, number of contigs and N50 [13,16,17]. Unfortunately, these measures are primitive and often misleading [20]. For example, N50, one of the most popular referencefree measures, can be maximized by trivial assemblies. N50 is defined as the length of the longest contig such that all contigs of at least that length compose at least $50 \%$ of the bases of the assembly [21]. The motivation for this measure is that better assemblies will result from a larger number of identified overlaps between the input 
reads and thus will have more reads assembled into longer contigs. However, it is easy to see that a trivial assembly constructed by concatenating all of the input reads into a single contig will maximize this measure. In short, N50 measures the continuity of contigs but not their accuracy [22]. Other simplistic reference-free measures can be similarly misleading regarding the accuracy of assemblies, although some have been shown to be potentially informative when assemblies include singletons (i.e., contigs derived from single reads) [20].

We improve upon the state-of-the-art in transcriptome assembly evaluation by presenting the DETONATE methodology ( $D E$ novo TranscriptOme rNa-seq Assembly with or without the Truth Evaluation) and software package. DETONATE consists of two components: RSEM-EVAL and REF-EVAL. RSEM-EVAL, DETONATE's primary contribution, is a reference-free evaluation method based on a novel probabilistic model that depends only on an assembly and the RNA-Seq reads used to construct it. RSEM-EVAL is similar to recent approaches using statistical models to evaluate or construct genome [23] and metagenome [24,25] assemblies, but, as we will discuss, is necessarily more complex because of widely varying abundances of transcripts and alternative splicing. Unlike simplistic reference-free measures such as N50, RSEM-EVAL combines multiple factors, including the compactness of an assembly and the support of the assembly from the RNA-Seq data, into a single, statistically principled evaluation score. This score can be used to select a best assembler, optimize an assembler's parameters and guide new assembler design as an objective function. In addition, for each contig within an assembly, RSEM-EVAL provides a score that assesses how well that contig is supported by the RNA-Seq data and can be used to filter unnecessary contigs. REF-EVAL, DETONATE's second component, is a toolkit of reference-based measures. It provides a more refined view of an assembly's accuracy than existing reference-based measures.

We have performed a number of experiments on both real and simulated data to demonstrate the value of the RSEM-EVAL score. First, we generated a set of perturbed assemblies around a single true assembly, and we show that RSEM-EVAL ranks the truth among the highest-scoring assemblies. Second, we computed RSEMEVAL scores and REF-EVAL reference-based measures on over 200 assemblies for multiple data sets, and we find that the RSEM-EVAL score generally correlates well with reference-based measures. The results of these first two experiments together show that the RSEM-EVAL score accurately evaluates de novo transcriptome assemblies, despite not having knowledge of the ground truth. Third, in comparison with several alternative reference-free and comparative-reference-based measures, we demonstrate the advantages of RSEM-EVAL in terms of accuracy, applicability and runtime requirements. Lastly, as a demonstration of the use of the RSEM-EVAL score, we assembled the transcriptome of the regenerating axolotl limb with its guidance. This new assembly allowed for the identification of many more genes that are involved in the process of axolotl limb regeneration than had been found with an assembly from a previous study.

\section{Results \\ DETONATE: a software package for the evaluation of de novo transcriptome assemblies}

The main contribution of our work is DETONATE, a methodology for the evaluation of de novo transcriptome assemblies and a software package that realizes this methodology. DETONATE consists of two components: RSEM-EVAL, which does not require a ground truth reference, and REF-EVAL, which does. The high-level workflow of DETONATE is shown in Figure 1. In the following subsections, we (1) describe the RSEM-EVAL reference-free score and show that the true assembly is an approximate local maximum of this score, (2) describe the REF-EVAL reference-based scores and show that RSEM-EVAL's score correlates well with these measures, indicating that RSEM-EVAL reflects the accuracy of a transcriptome assembly, and (3) demonstrate our methods' practical usefulness by assembling the transcriptome of the regenerating axolotl limb with the guidance of RSEM-EVAL. To understand the components of DETONATE and the experiments we have performed with them best, we first define

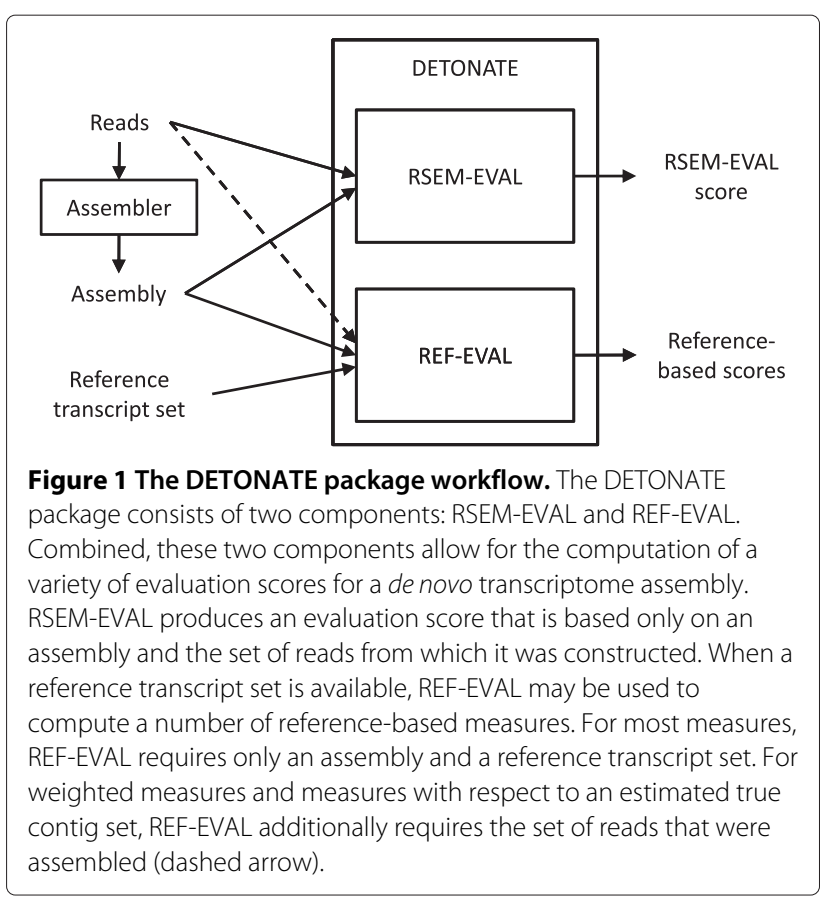


what is considered to be the true assembly of a set of RNA-Seq reads, which is critical to the assembly evaluation task.

\section{The true assembly according to DETONATE}

Ideally, the goal of transcriptome assembly would be to construct the full-length sequences of all transcripts in the transcriptome. Unfortunately, it is rarely possible to achieve this goal in practice, because sequencing depths are usually not high enough to cover all transcripts completely, especially those of low abundance. Thus, a transcriptome assembly is, in general, a set of contigs, with each contig representing the sequence of a segment of a transcript. When paired-end data are assembled, one can also consider constructing scaffolds, or chains of contigs separated by unknown sequences with estimated lengths.

Both RSEM-EVAL and REF-EVAL make use of the concept of the true assembly of a set of RNA-Seq reads, which is the assembly one would construct if given knowledge of the true origin of each read. A precise definition of the true assembly is provided in the Materials and methods. Intuitively, for each non-negative integer $w$, the true contig assembly at minimum overlap length $w$ is the collection of transcript subsequences that are covered by reads whose true alignments overlap by at least $w$ bases. The true contig assembly at minimum overlap length 0 is the collection of transcript subsequences covered by reads whose true alignments are contiguous (that is, overlap by zero bases) or overlap by at least one base. The true contig assembly at minimum overlap length 0 is the best theoretically achievable contig assembly in that it represents all supported segments of the transcript sequences, and no unsupported segments. Figure 2 gives an example of true contig assemblies at minimum overlap lengths $w=0$ and $w=1$. With paired-end data, the true assembly is the set of scaffolds that one would obtain by scaffolding the true contig

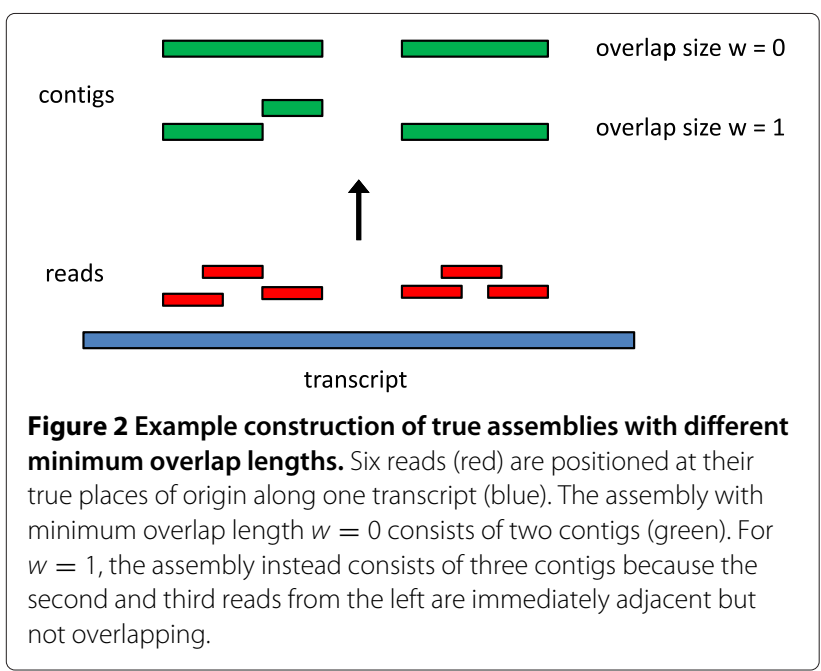

assembly with complete knowledge of which contigs are linked by a read pair and the distances between contigs.

\section{RSEM-EVAL is a novel reference-free transcriptome assembly evaluation measure}

RSEM-EVAL, our primary contribution, is a referencefree evaluation measure based on a novel probabilistic model that depends only on an assembly and the RNASeq reads used to construct it. In short, the RSEM-EVAL score of an assembly is defined as the log joint probability of the assembly $A$ and the reads $D$ used to construct it, under a model that we have devised. In symbols:

$$
\operatorname{score}_{\text {RSEM-EVAL }}(A)=\log P(A, D)
$$

RSEM-EVAL's intended use is to compare several assemblies of the same set of reads, and under this scenario, the joint probability is proportional to the posterior probability of the assembly given the reads. Although the posterior probability of the assembly given the reads is a more natural measure for this application, we use the joint probability because it is more efficient to compute.

The details of the RSEM-EVAL model are provided in the Materials and methods. In summary, the RSEMEVAL score consists of three components: a likelihood, an assembly prior and a Bayesian information criterion (BIC) penalty. That is:

$$
\begin{aligned}
\log P(A, D)= & \log \int_{\Lambda} P(D \mid A, \Lambda) P(A \mid \Lambda) P(\Lambda) d \Lambda \\
\approx & \underbrace{\log P\left(D \mid A, \Lambda_{\mathrm{MLE}}\right)}_{\text {likelihood }}+\underbrace{\log P\left(A \mid \Lambda_{\mathrm{MLE}}\right)}_{\text {assembly prior }} \\
& -\underbrace{\frac{1}{2}(M+1) \log N}_{\text {BIC penalty }}
\end{aligned}
$$

where $N$ is the total number of reads (or read pairs, for paired-end data), $M$ is the number of contigs (scaffolds) in the assembly, and $\Lambda_{\mathrm{MLE}}$ is the maximum likelihood (ML) estimate of the expected read coverage under $P(A, D \mid \Lambda)$. For typical sizes of RNA-Seq data sets used for transcriptome assembly, the likelihood is generally the dominant component of the RSEM-EVAL score in the above equation. It serves to assess how well the assembly explains the RNA-Seq reads. However, as we will show later, only having this component is not enough. Thus we use the assembly prior and BIC components to assess an assembly's complexity. These two components penalize assemblies with too many bases or contigs (scaffolds), or with an unusual distribution of contig (scaffold) lengths relative to the expected read coverage. Thus, these two components impose a parsimony preference on the RSEM-EVAL score. The three components together enable the RSEM-EVAL score to favor simple assemblies that can explain the RNA-Seq reads well. 


\section{The ground truth is an approximate local maximum of the RSEM-EVAL score}

As we have discussed, the true assembly at minimum overlap length 0 is the best possible assembly that can be constructed solely from RNA-Seq data. Therefore, we consider it to be the ground truth assembly. A good evaluation measure should score the ground truth among its best assemblies. Ideally, we would have explored the entire space of assemblies and shown that the RSEM-EVAL score for the ground truth assembly is among the highest scores. However, such a global search of assembly space is computationally infeasible. Thus, we instead performed experiments that assess whether in the local space around the ground truth, the ground truth is among the best scoring assemblies. In other words, we tested whether the ground truth assembly is approximately a local maximum of the RSEM-EVAL function.

We explored the local space of assemblies around that of the ground truth by generating assemblies that were slightly perturbed from it. We performed experiments with two kinds of perturbations: random perturbations and guided perturbations. In our random perturbation experiments, assemblies were generated by randomly mutating the ground truth assembly. Since the minimum overlap length is a critical parameter for constructing assemblies, we also assessed the RSEM-EVAL scores for true assemblies with different minimum overlap lengths in guided perturbation experiments. A good evaluation score should generally prefer true assemblies with small minimum overlap lengths, which are closest to the ground truth.

For these experiments, it was critical that the ground truth assembly be known, and therefore we primarily used a simulated set of RNA-Seq data, in which the true origin of each read is known. In addition, for our guided perturbation experiments, we used the real mouse data set on which the simulated data were based, and we estimated the true origin of each read. For details about these real and simulated data, see the Materials and methods.

\section{Random perturbation}

With our random perturbation experiment we wished to determine how well, in terms of the RSEM-EVAL score, the ground truth compares to assemblies in the local space surrounding it. To explore the space of assemblies centered at the ground truth thoroughly, we used four types of mutations (substitution, fusion, fission and indels), each of which was applied at five different strength levels (see Materials and methods). Therefore, in total, we generated 20 classes of randomly perturbed assemblies. For each class, we generated 1,000 independent randomly perturbed assemblies to estimate the RSEM-EVAL score population mean and its $95 \%$ confidence interval for assemblies of that class.
On average, the perturbed assemblies had RSEM-EVAL scores that were worse than that of the ground truth (Figure 3A). In addition, the higher the mutation strength, the worse the mean score of the perturbed assemblies. This suggests that the ground truth assembly behaves similarly to a local maximum of the RSEM-EVAL function. Even though the population mean scores of the perturbed assemblies were estimated to be always worse than the score of the ground truth, individual perturbed assemblies could have had higher scores. Therefore, for each class of perturbed assemblies, we calculated the fraction of assemblies with RSEM-EVAL scores larger than that of the ground truth, which we refer to as the error rate. Error rates decreased dramatically with increasing mutation strength and, for all mutation types except fusion, error rates were only non-zero for the weakest mutation strength level (Figure 3B). RSEM-EVAL had the most trouble with the fusion-perturbed assemblies, with more than half of such assemblies at the weakest mutation strength having a score above that of the ground truth. From individual examinations of these assemblies, we observed that in many of these cases, the assemblies contained fusions of contigs with low abundances, which are difficult to distinguish from true contigs, especially with the ground truth defined as the true assembly with minimum overlap length $w=0$.

\section{Guided perturbation}

With the guided perturbation experiments, we measured the RSEM-EVAL scores of assemblies constructed with different values of the minimum overlap length, which is a common parameter in assemblers. Since the true assembly at minimum overlap length 0 is the best achievable assembly, a good evaluation score should be maximized at small minimum overlap lengths. As described before, we used one simulated and one real mouse RNA-Seq data set for these experiments. For each data set, we constructed the true assemblies with minimum overlap lengths ranging from 0 to 75 . The true assembly at minimum overlap length 76 (the read length) was not constructed because of prohibitive running times. For the real RNA-Seq data, true assemblies were estimated using REF-EVAL's procedure, described below. We then calculated the RSEM-EVAL scores for all of these assemblies.

As we had hoped, we found that the RSEM-EVAL score was maximized at small minimum overlap lengths, for both the simulated and real data sets (Figure 4). In contrast, the ML score increased with minimum overlap length and peaked at minimum overlap length $w=75$. These results support the necessity of the prior of the RSEM-EVAL model, which takes into account the complexity of an assembly.

To explore the effects of the minimum overlap length parameter, $w$, in the RSEM-EVAL model, we also per- 


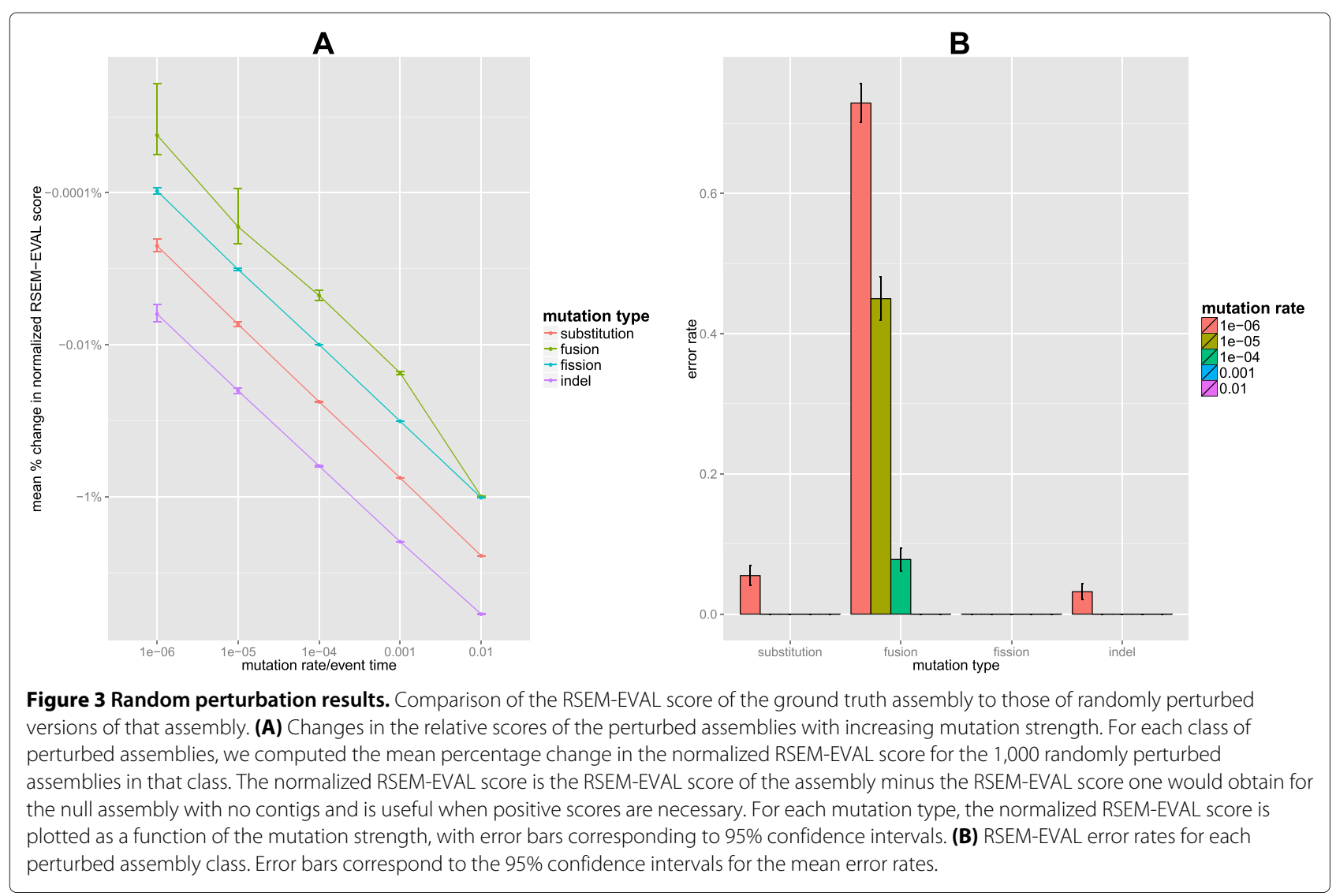

formed the guided perturbation experiments with $w=50$ for the RSEM-EVAL model. We did not observe any major differences between these results and those for $w=0$ (Additional file 1: Figures S3 and S4). To explain this result, note that although the RSEM-EVAL model uses $w$ in both the prior and likelihood correction components, our estimation procedure for the uncorrected likelihood component (see Materials and methods) does not explicitly check for violations of the minimum overlap length by the assembly (i.e., regions that are not covered by reads that overlap each other by at least $w$ bases). Thus, the minimum overlap length does not play a role in the uncorrected likelihood, which is the dominant term of the RSEM-EVAL score.

\section{REF-EVAL is a refined toolset for computing reference-based evaluation measures}

Our first experiment, above, shows that RSEM-EVAL has an approximate local maximum at the true assembly. However, this does not necessarily imply that RSEMEVAL induces a useful ranking of assemblies away from this local maximum. Thus, to assess the usefulness of RSEM-EVAL's reference-free score, it is of interest to compare the ranking RSEM-EVAL assigns to a collection of assemblies to the ranking assigned by comparing each assembly to a reference. This raises two questions: (1) What reference do we compare against? (2) How do we perform the comparison? REF-EVAL constitutes an answer to both questions. The tools REF-EVAL provides are also of independent interest for reference-based evaluation of transcriptome assemblies.

In answer to question (1), REF-EVAL provides a method to estimate the true assembly of a set of reads, relative to a collection of full-length reference transcript sequences. The estimate is based on alignments of reads to reference transcripts, as described in the Materials and methods. As we have previously discussed, we wish to compare assemblies against the set of true contigs or scaffolds instead of full-length reference sequences because the latter cannot, in general, be fully reconstructed from the data and we want to reward assemblies for recovering read-supported subsequences of the references.

In answer to question (2), REF-EVAL provides two kinds of reference-based measures. First, REF-EVAL provides assembly recall, precision, and $F_{1}$ scores at two different granularities: contig (scaffold) and nucleotide. Recall is the fraction of reference elements (contigs, scaffolds or nucleotides) that are correctly recovered by an assembly. Precision is the fraction of assembly elements that correctly recover a reference element. The $F_{1}$ score is 

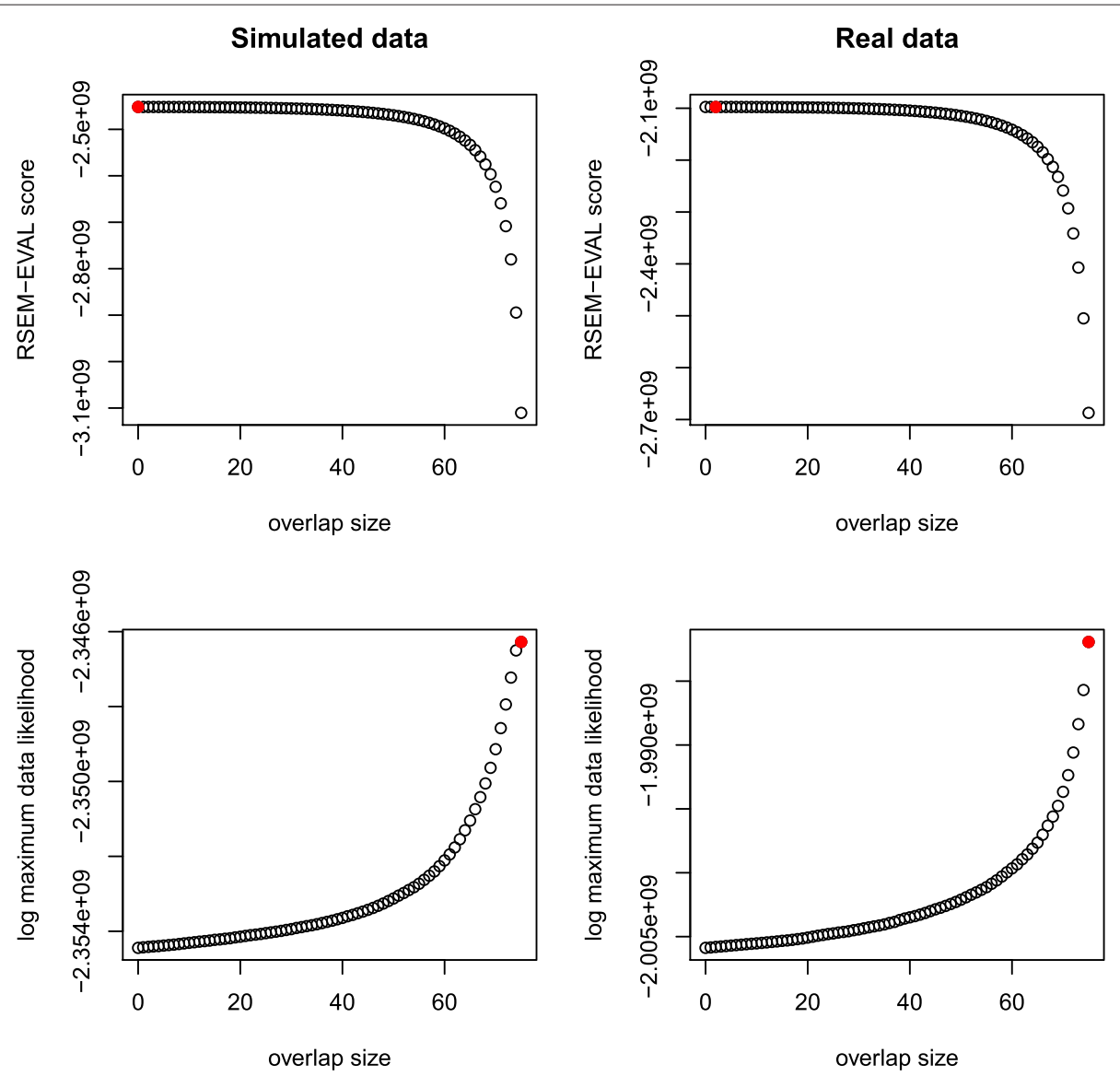

Figure 4 Guided perturbation results. RSEM-EVAL (top row) and maximum likelihood (bottom row) scores of true assemblies for different values of the minimum overlap length $w$ on both simulated (left column) and real (right column) data sets. The maximizing values (red circles) are achieved at $w=0, w=2, w=75$ and $w=75$ in a top-down, left-right order. For better visualization of the maximizing values of $w$, RSEM-EVAL scores for the local regions around the maximal values are shown in Additional file 1: Figure S2.

the harmonic mean of recall and precision. For precise definitions and computational details, see Materials and methods.

Although the contig- (scaffold-) and nucleotide-level measures are straightforward and intuitive, both have drawbacks and the two measures can be quite dissimilar (Figure 5). For example, if two contigs align perfectly to a single reference sequence, but neither covers at least $99 \%$ of that sequence, the nucleotide-level measure will count them as correct, whereas the contig-level measure will not (Figure 5B). In general, the contigand scaffold-level measurements can fail to give a fair assessment of an assembly's overall quality, since they use very stringent criteria and normally only a small fraction of the reference sequences are correctly recovered. And whereas the nucleotide-level measure arguably gives a more detailed picture of an assembly's quality, it fails to take into account connectivity between nucleotides. For example, in the example depicted in Figure 5B, the nucleotide-level measure does not take into account the fact that the correctly predicted nucleotides

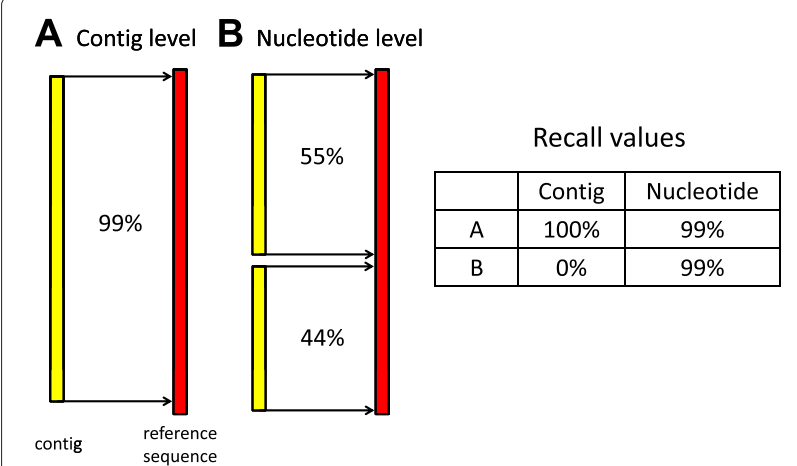

Figure 5 Different granularities of reference-based measures computed by REF-EVAL. (A) The contig-level measure requires at least 99\% alignment between a matched contig and reference sequence in a one-to-one mapping between an assembly and the reference. (B) The nucleotide-level measure counts the number of correctly recovered nucleotides without requiring a one-to-one mapping. Unlike the contig-level measure, it gives full credit to the two short contigs. The table on the right gives both the contig-level and nucleotide-level recall values for (A) and (B). 
of the reference sequence are predicted by two different contigs.

Motivated, in part, by the shortcomings of the contig-, scaffold-, and nucleotide-level measures, REF-EVAL also provides a novel transcriptome assembly reference-based accuracy measure, the $k$-mer compression score (KC score). In devising the $\mathrm{KC}$ score, our goals were to define a measure that would (1) address some of the limitations of the other measures, (2) provide further intuition for what the RSEM-EVAL score optimizes and (3) be relatively simple. The $\mathrm{KC}$ score is a combination of two measures, weighted $k$-mer recall (WKR) and inverse compression rate (ICR), and is simply defined as:

$$
\text { score }_{\mathrm{KC}}=\mathrm{WKR}-\mathrm{ICR}
$$

WKR measures the fidelity with which a particular assembly represents the $k$-mer content of the reference sequences. Balancing WKR, ICR measures the degree to which the assembly compresses the RNA-Seq data. WKR and ICR are defined and further motivated in the Materials and methods.

\section{The RSEM-EVAL score correlates highly with reference-based measures}

Having specified a framework for reference-based transcriptome assembly evaluation via REF-EVAL, we then sought to test whether the RSEM-EVAL score ranks assemblies similarly to REF-EVAL's reference-based measures. To test this, we constructed a large number of assemblies on several RNA-Seq data sets from organisms for which reference transcript sequences were available, and we computed both the RSEM-EVAL score and reference-based measures for each assembly. The RNASeq data sets used were the simulated and real mouse strand non-specific data from the perturbation experiments, a real strand-specific mouse data set and a real strand-specific yeast data set. Four publicly available assemblers, Trinity [4], Oases [6], SOAPdenovo-Trans [8] and Trans-ABySS [2], were applied to assemble these data sets using a wide variety of parameter settings.

\section{Overall correlation}

For each data set, we computed Spearman's rank correlation between the reference-based measure values and the RSEM-EVAL scores to measure the similarity of the rankings implied by them. For single-end data, RSEMEVAL scores had decent correlation with the contig and nucleotide-level $F_{1}$ measures on the strand non-specific (Figure 6) and strand-specific (Additional file 1: Figure S5) data sets. Specifically, the correlation of the contig and nucleotide-level $F_{1}$ measures to the RSEM-EVAL score is comparable to the correlation of the contig and nucleotide-level $F_{1}$ measures to each other. RSEM-EVAL performed similarly well on the paired-end strand nonspecific data set (Additional file 1: Figure S6).

The RSEM-EVAL scores had markedly higher correlations with the $\mathrm{KC}$ score ( $k=L$, the read length) for both the strand non-specific (Figure 7) and strand-specific (Additional file 1: Figure S7) single-end data sets, as well as for the paired-end data set (Additional file 1: Figure S6), which confirmed our expectations given the mathematical connections between these scores. To assess the impact of the $k$-mer size on the KC score, we also computed correlations between the RSEM-EVAL score and the KC score at half $(k=36)$ and double $(k=152)$ the read length for the strand non-specific single-end data. We found that these correlation values were not sensitive to the value of $k$ (Additional file 1: Figures S8 and S9). These results provide some intuition for what the RSEM-EVAL score assesses and indicate that the RSEM-EVAL score could be used as a proxy for the $\mathrm{KC}$ score when reference sequences are not known.

Although this experiment was not designed as a comprehensive evaluation, some features of these results are suggestive of the relative accuracies of the assemblers. First, given the selected assembler versions and parameter settings, Trinity produced the most accurate assemblies for all data sets with respect to the contig-, scaffoldand nucleotide-level $F_{1}$ scores and the $\mathrm{KC}$ score. The RSEM-EVAL score supported this result, with the Trinity assemblies also obtaining the highest RSEM-EVAL scores. Second, varying the Trinity parameters had a relatively small effect on the accuracy of the resulting assemblies, compared to Oases and SOAPdenovo-Trans, which produced assemblies that spanned a large range of accuracies. From the assemblies of the mouse strand non-specific single-end data produced by the assemblers with their default parameters, we identified a case that exemplifies Trinity's accuracy and demonstrates how RSEM-EVAL selects the best assembly (Figure 8).

\section{Comparison to other measures}

As we mentioned in the introduction, there are a wide variety of other measures that have been proposed and used for the evaluation of assemblies. We selected a representative set of such measures for comparison with RSEM-EVAL. From the simple reference-free measures, we selected N50 because of its popularity and the number of bases in (non-singleton) contigs because this measure was determined to be 'strong' and 'fully consistent' for evaluating de novo transcriptome assemblies [20]. Genovo [24] and ALE [25] both provide model-based referencefree scores for evaluating metagenome assemblies, which are highly similar to transcriptome assemblies, and thus we also included these scores for comparison. Lastly, we compared RSEM-EVAL to two comparativereference-based measures that may be used if a protein 


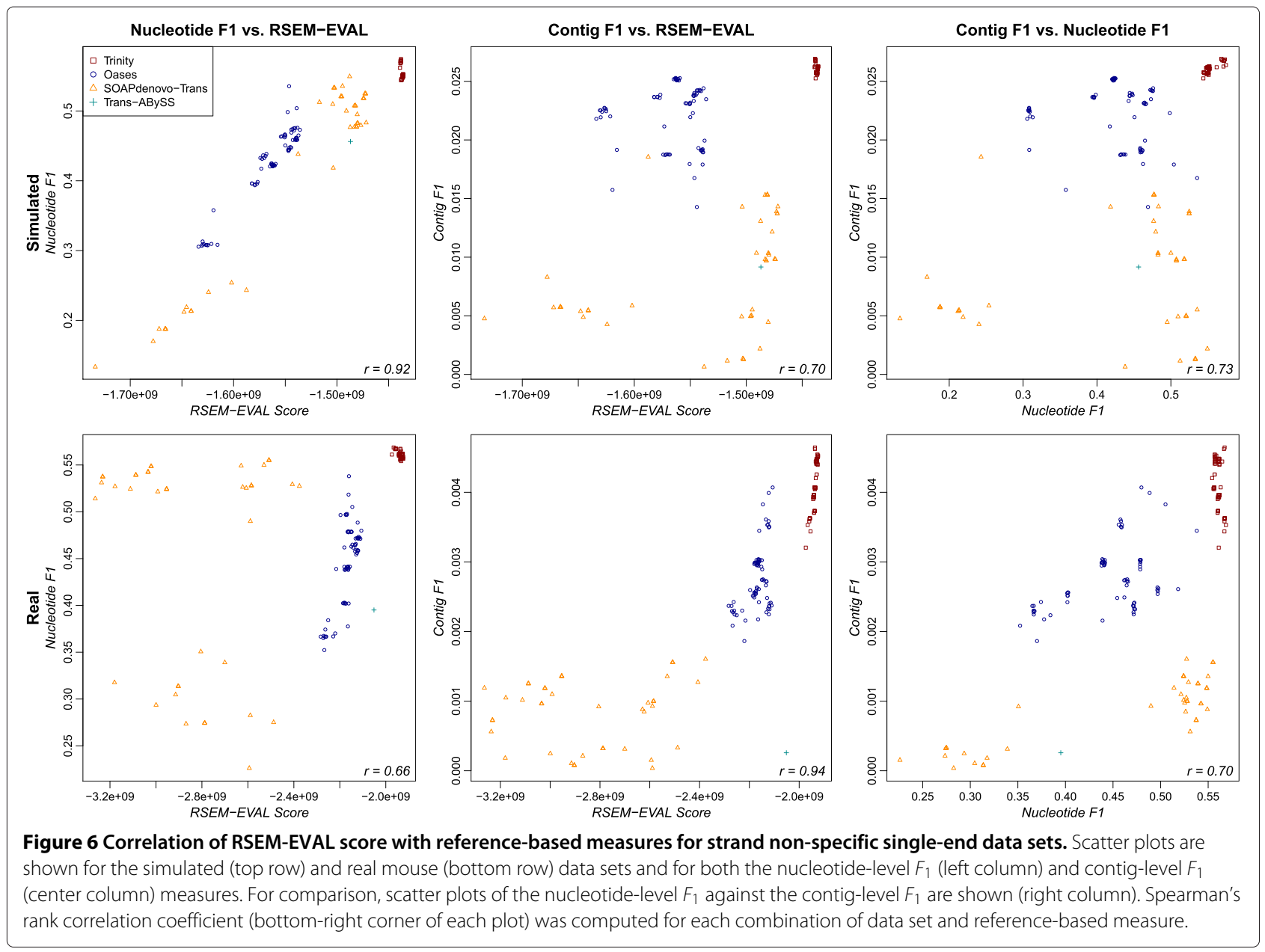

set from a closely related species is available: the ortholog hit ratio [26] and the number of unique proteins matched by assembly elements, both of which were determined to be effective for transcriptome assembly evaluation [20].
Because some of these measures were computationally costly to compute, we evaluated them with a smaller data set than that used in the previous section. Specifically, we used the set of reads from the real mouse strand non-specific single-end data set that mapped to genes on
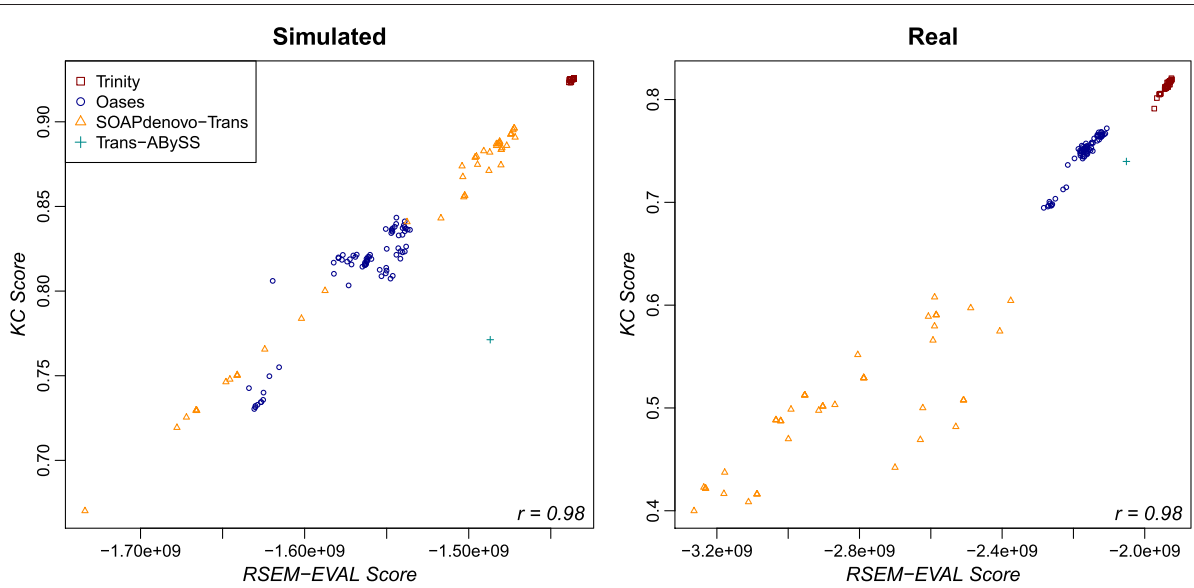

Figure 7 Correlation of the RSEM-EVAL and KC scores on the strand non-specific single-end data sets. Spearman's rank correlation coefficient (bottom-right corner of each plot) was computed for each data set. KC score, $k$-mer compression score. 


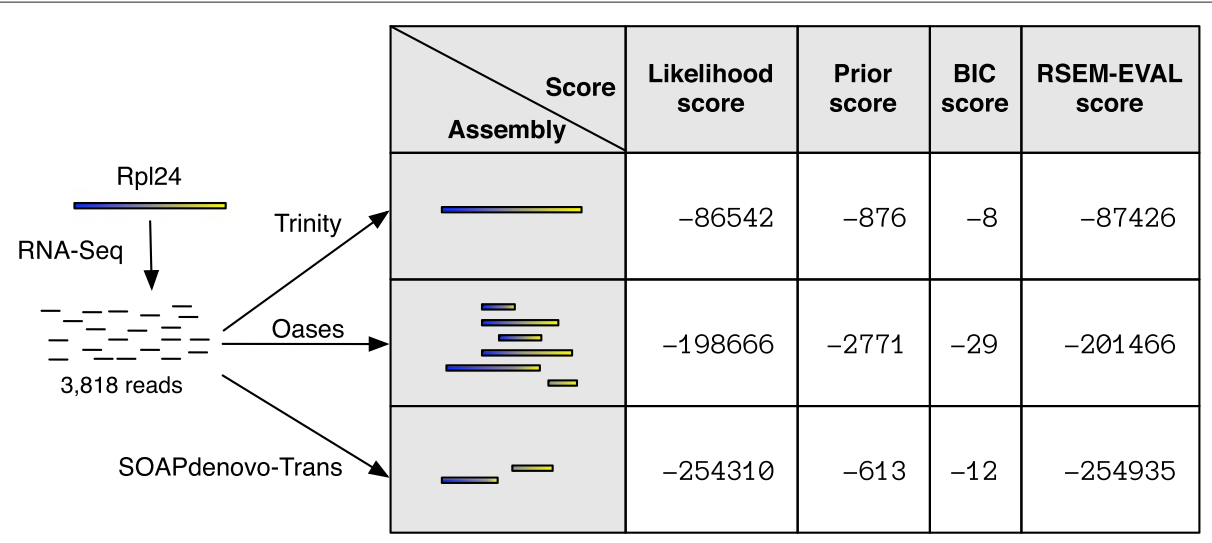

Figure 8 RSEM-EVAL correctly selects the Trinity assembly of reads originating from a transcript of mouse gene Rp/24 as the best among the default assemblies from Trinity, Oases and SOAPdenovo-Trans. Reads from the mouse strand non-specific single-end data set aligning to transcript 1 of Rpl24 were extracted and assembled by Trinity, Oases and SOAPdenovo-Trans with default parameters. Contigs (filled rectangles) from each assembly were aligned against the true transcript with BLAT to establish their positional identities (blue-yellow fill, with only the segment of a contig from its highest-scoring local alignment shown). RSEM-EVAL was run on each assembly and the likelihood, prior, BIC and total RSEM-EVAL scores were recorded. Although the SOAPdenovo-Trans assembly was smaller (as reflected by the higher prior score), the Trinity assembly had a much higher likelihood score, which is generally the dominant term in the RSEM-EVAL score, and was thus correctly selected as the most accurate assembly. BIC, Bayesian information criterion.

chromosome 1. As in the previous section, we assembled these reads with a variety of assemblers and parameter settings and computed the selected set of measures along with the RSEM-EVAL and REF-EVAL measures on the resulting assemblies.

In terms of Spearman's rank correlation, RSEM-EVAL outperformed all other measures with respect to the contig-level $F_{1}$ and $\mathrm{KC}$ scores, but had lower correlation with the nucleotide-level $F_{1}$ score than the Genovo, ALE and number of unique proteins matched measures (Table 1, Additional file 1: Figures S10 and S11).

Table 1 Spearman's rank correlation coefficient of the scores assigned by several alternative transcriptome assembly evaluation measures to the reference-based scores from REF-EVAL

\begin{tabular}{llll}
\hline & KC score & Contig F1 & Nucleotide F1 \\
\hline RSEM-EVAL score & 0.99 & 0.83 & 0.46 \\
Genovo score & 0.96 & 0.80 & 0.53 \\
ALE score & 0.64 & 0.45 & 0.62 \\
N50 & 0.22 & 0.33 & -0.31 \\
Number of nucleotides & 0.13 & 0.29 & -0.21 \\
in assembly & & & \\
Number of unique proteins & 0.68 & 0.81 & 0.73 \\
matched & & & -0.19 \\
Average ortholog hit ratio & 0.31 & 0.31 & \\
\hline
\end{tabular}

The alternative measures are defined in the main text. The evaluated assemblies were produced by Trinity, Oases, SOAPdenovo-Trans and Trans-ABySS, based on the subset of reads in the real (strand non-specific) mouse data that align to genes on chromosome 1. This subset was used in the interest of computational efficiency of the alternative measures.

KC score, $k$-mer compression score.
RSEM-EVAL, Genovo and ALE were the only referencefree measures to have positive correlations with respect to all three REF-EVAL measures. N50 and the number of bases in contigs measures had negative correlation with the nucleotide-level $F_{1}$ score and positive but poor correlation with the other REF-EVAL measures. Unsurprisingly, because of the similarity of the models used by RSEM-EVAL and Genovo, the scores produced by the two methods were also similar (Additional file 1: Figure $\mathrm{S} 10)$. Although ALE is also a model-based reference-free measure, it had noticeably different behavior from RSEMEVAL and Genovo, particularly for the Oases assemblies, which were generally larger than the other assemblies. Of the two comparative-reference-based measures, the number of unique proteins matched measure performed best, achieving good correlation with all REF-EVAL measures and the highest correlation (0.73) with the nucleotidelevel $F_{1}$ score. The ortholog hit ratio measure did not fare as well and, in fact, had negative correlation with the nucleotide-level $F_{1}$ score.

Given the similarity of RSEM-EVAL to Genovo and ALE, both in terms of their underlying methodology and their performance on the selected data set, we sought to differentiate RSEM-EVAL further from these methods. First, we note that unlike RSEM-EVAL, the Genovo and ALE scores do not explicitly take into account transcript abundances and only use one alignment per read, even if a read has multiple equally good alignments. To demonstrate the necessity of modeling transcript abundance and read mapping uncertainty, we constructed a simple realistic example in which only RSEM-EVAL correctly scores the true assembly as the best (Figure 9). 


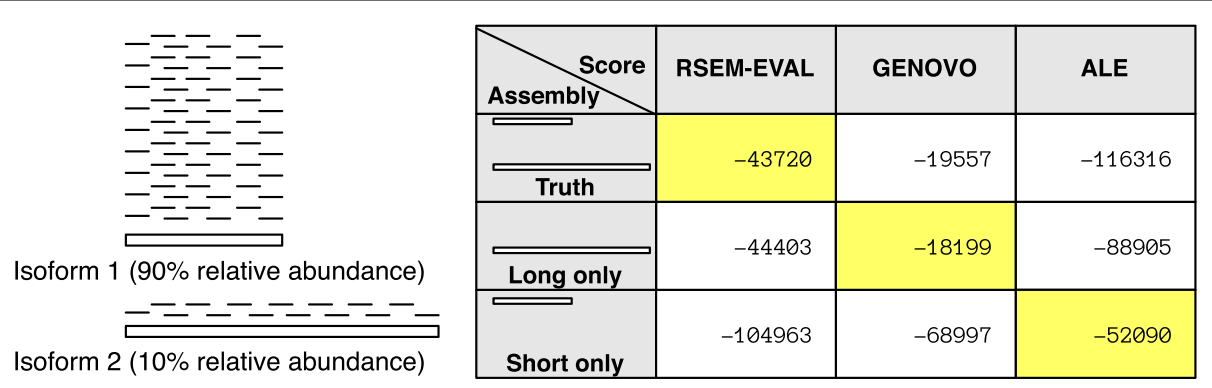

Figure 9 Example scenario in which RSEM-EVAL correctly selects the true assembly whereas Genovo and ALE select suboptimal assemblies. Because Genovo and ALE do not explicitly take into account transcript abundance and read mapping uncertainty, scenarios in which multiple isoforms of the same gene are present in an RNA-Seq sample can confuse these methods. In this example, a gene has two isoforms, the first isoform (with a length of 1,000 bases) corresponding to the first half of the second isoform (with a length of 2,000 bases). We simulated 5,000 single-end RNA-Seq reads of length 100 bases with $0.01 \%$ sequencing error from these transcripts and with a $90: 10$ abundance ratio between the first and second isoforms, respectively. Because RSEM-EVAL models transcript abundances and takes into account read mapping uncertainty, it correctly scores the true assembly the highest. In contrast, Genovo selects the assembly containing only the long isoform and ALE selects the assembly containing only the short isoform.

Second, we measured the runtime and memory usage of each of these software packages on the full mouse strand non-specific single-end data set and found that RSEMEVAL is substantially faster than both Genovo and ALE, which have arguably prohibitive runtimes for this realistic data set (Table 2). Lastly, RSEM-EVAL and ALE have richer models than Genovo, both supporting paired-end data, quality scores and strand specificity.

\section{Within-assembler correlation}

One important potential application of RSEM-EVAL is the optimization of the parameters of an assembler. Thus, it is of interest whether the RSEM-EVAL score correlates well with reference-based measures for assemblies generated by a single assembler. In a previous subsection, we showed that the RSEM-EVAL score has high correlation with the KC score for real and simulated data when several different assemblers are used. Looking at each assembler separately, we also find that the RSEM-EVAL score has high correlation with the $\mathrm{KC}$ score when only the assembler's parameters are changed, for both strand nonspecific (Figure 10) and strand-specific (Additional file 1: Figure S12) single-end data sets, as well as for the pairedend data set (Additional file 1: Figure S13). This suggests that RSEM-EVAL can be used to optimize the parameters of an assembler for a given data set when the KC score is of interest for measuring the accuracy of an assembly.

\section{Assessing the relative impact of individual contigs or scaffolds within an assembly}

The RSEM-EVAL score is an assembly-level measure that allows one to compare different assemblies constructed

Table 2 Wall-clock runtimes (in hours, minutes and seconds) and memory usage (as measured by the maximum resident set size, in gigabytes) for several assembly evaluation tools

\begin{tabular}{|c|c|c|c|c|c|c|}
\hline \multirow[b]{2}{*}{ Program } & \multicolumn{2}{|c|}{ Assembly T } & \multicolumn{2}{|c|}{ Assembly 0} & \multicolumn{2}{|c|}{ Assembly S } \\
\hline & Runtime & Memory & Runtime & Memory & Runtime & Memory \\
\hline RSEM-EVAL ${ }^{a}$ & $1 \mathrm{~h} 4 \mathrm{~m} 57 \mathrm{~s}$ & $2.02 \mathrm{~GB}$ & $4 \mathrm{~h} 40 \mathrm{~m} 36 \mathrm{~s}$ & $8.18 \mathrm{~GB}$ & $34 \mathrm{~m} 57 \mathrm{~s}$ & $1.23 \mathrm{~GB}$ \\
\hline Genovo & $6 \mathrm{~d} 11 \mathrm{~h} 54 \mathrm{~m} \mathrm{3s}$ & 192.23 GB & $>1$ week & - & $4 \mathrm{~d} 15 \mathrm{~h} 3 \mathrm{~m} 3 \mathrm{~s}$ & $188.79 \mathrm{~GB}$ \\
\hline $\mathrm{ALE}^{\mathrm{a}}$ & 12 h 39 m 36 s & $0.67 \mathrm{~GB}$ & $6 \mathrm{~d} 23 \mathrm{~h} 23 \mathrm{~m} 13 \mathrm{~s}$ & $2.31 \mathrm{~GB}$ & $7 \mathrm{~h} 33 \mathrm{~m} 1 \mathrm{~s}$ & $0.59 \mathrm{~GB}$ \\
\hline REF-EVAL, contig ${ }^{b}$ & $3 s$ & $0.19 \mathrm{~GB}$ & $8 s$ & $0.33 \mathrm{~GB}$ & $2 \mathrm{~s}$ & $0.2 \mathrm{~GB}$ \\
\hline REF-EVAL, nucleotide ${ }^{b}$ & $8 s$ & $0.39 \mathrm{~GB}$ & $33 s$ & $1.27 \mathrm{~GB}$ & $6 s$ & $0.33 \mathrm{~GB}$ \\
\hline REF-EVAL, KC score & $1 \mathrm{~m} 18 \mathrm{~s}$ & $2.09 \mathrm{~GB}$ & $1 \mathrm{~m} 30 \mathrm{~s}$ & $2.37 \mathrm{~GB}$ & $1 \mathrm{~m} 13 \mathrm{~s}$ & $2.03 \mathrm{~GB}$ \\
\hline Bowtie & $15 \mathrm{~m} \mathrm{42s}$ & $0.11 \mathrm{~GB}$ & $1 \mathrm{~h} 1 \mathrm{~m} 38 \mathrm{~s}$ & $0.31 \mathrm{~GB}$ & $11 \mathrm{~m} 16 \mathrm{~s}$ & $0.1 \mathrm{~GB}$ \\
\hline BLAT & $35 \mathrm{~m} \mathrm{14s}$ & $0.0 \mathrm{~GB}$ & $1 \mathrm{~h} 51 \mathrm{~m} 1 \mathrm{~s}$ & $0.01 \mathrm{~GB}$ & $28 \mathrm{~m} \mathrm{19s}$ & $0.0 \mathrm{~GB}$ \\
\hline
\end{tabular}

a Plus time to run Bowtie. We calculate Bowtie statistics separately because ALE takes Bowtie alignments as input.

bPlus time to run BLAT.

Each tool was run on three different assemblies of the real mouse data. Assembly T was produced by Trinity with its default parameters ( 52,667 contigs, 33 million nucleotides). Assembly $\mathrm{O}$ was produced by Oases with its default parameters (160,455 contigs, 115 million nucleotides). Assembly $\mathrm{S}$ was produced by SOAPdenovo-Trans with its default parameters (79,460 contigs, 28 million nucleotides). Multithreaded programs (RSEM-EVAL, REF-EVAL and Bowtie) were run with 16 threads. All programs were run on a compute server with an Intel ${ }^{\circledR}$ Xeon ${ }^{\oplus}$ CPU E5-2660 v2 $2.20 \mathrm{GHz}$ processor and 500 gigabytes RAM. Genovo had not finished running on assembly $\mathrm{O}$ after more than 1 week. KC score, $k$-mer compression score. 


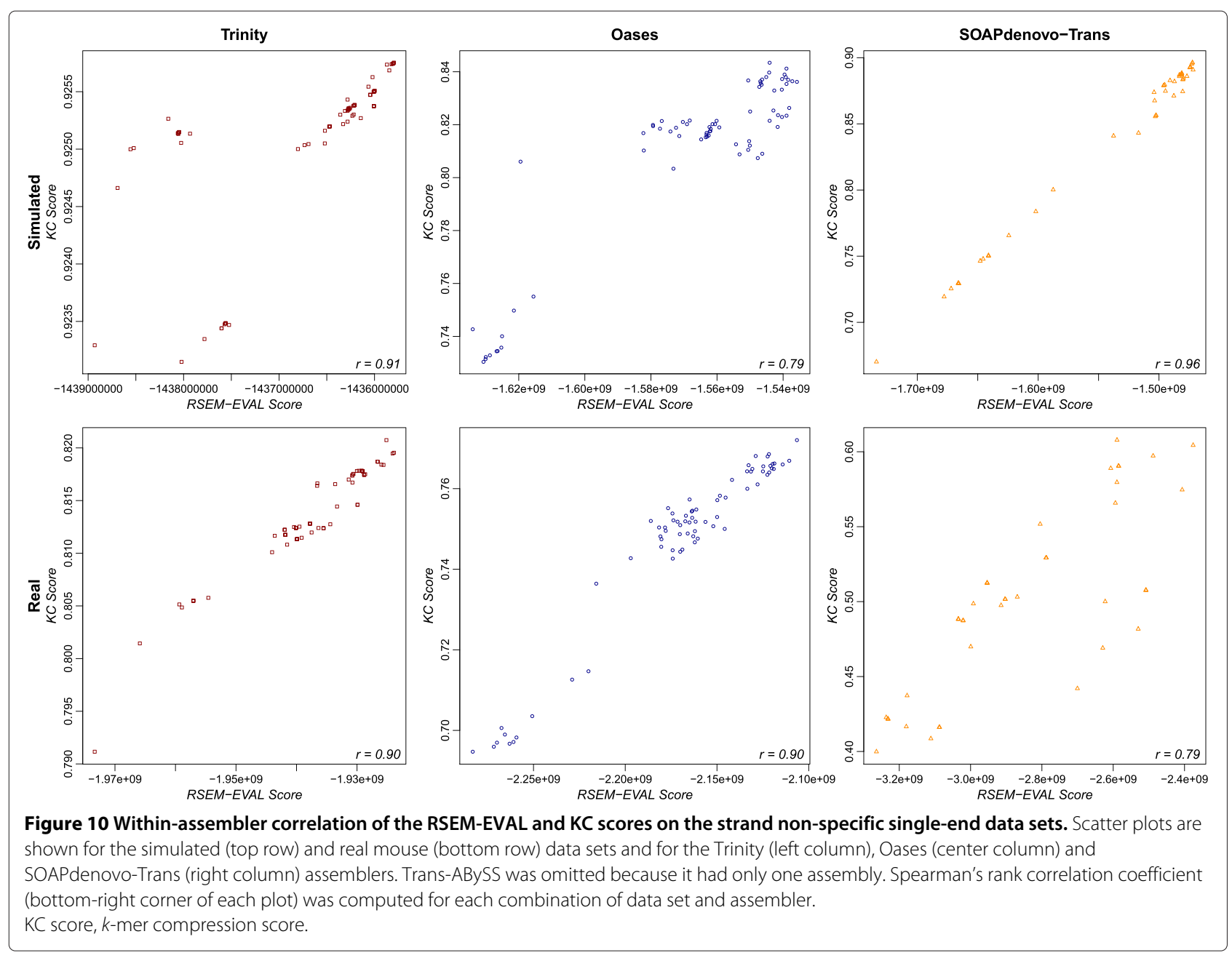

from the same data set. It is also of interest to compute scores for individual contigs or scaffolds within an assembly that reflect their relative impacts. In this section we describe and assess a contig-level score based on RSEMEVAL for single-end data. RSEM-EVAL can analogously produce scaffold-level scores when paired-end data are available.

One natural statistical approach for assessing the explanatory power of a contig is to compare the hypothesis that a particular contig is a true contig with the null hypothesis that the reads composing the contig are actually from the background noise. For each contig, we use the $\log$ of the ratio between the probabilities for these two hypotheses as its contig impact score. Through a decomposition of the RSEM-EVAL score $\log P(A, D)$ into contig-level components, we are able to calculate these contig impact scores efficiently (Additional file 1 : Section 5).

RSEM-EVAL's contig impact score measures the relative contribution of each contig to explaining the assembled RNA-Seq data. This suggests a strategy to improve the accuracy of an assembly: trim it by removing contigs that contribute little to explaining the data. To evaluate this strategy (and by extension the contig impact score itself), we trimmed assemblies of the simulated data using the RSEM-EVAL contig impact scores and computed the resulting changes in the evaluation measures. Assemblies were trimmed by removing all contigs with negative scores.

In general, the trimmed assemblies had better evaluation scores than their untrimmed counterparts (Additional file 1: Table S2 and Figure S14). The largest improvements were seen for assemblies produced by Oases and Trans-ABySS, which tend to produce large numbers of contigs. In fact, for both the nucleotideand contig-level $F_{1}$ scores, the trimmed Oases assemblies were the most accurate of all assemblies (both trimmed and untrimmed), supporting the usefulness of the RSEM-EVAL contig impact score. This suggests that the RSEM-EVAL contig impact scores are correctly identifying contigs that are either erroneous or redundant within these assemblies. 


\section{RSEM-EVAL guides creation of an improved axolotl assembly}

The axolotl (Ambystoma mexicanum) is a neotenic salamander with regenerative abilities that have piqued the interests of scientists. In particular, there is significant interest in studying the molecular basis of axolotl limb regeneration [27]. Although the axolotl is an important model organism, its genome is large and repetitive, and, as a result, it has not yet been sequenced. In addition, a publicly available, complete and high-quality set of axolotl transcript sequences does not exist, which makes it challenging to study the axolotl's transcriptome.

To demonstrate the use of RSEM-EVAL, we employed it to select an assembler and parameter values for a set of previously published RNA-Seq data from a time-course study of the regenerating axolotl limb blastema [27]. This data set consisted of samples taken at 0 hours, 3 hours, 6 hours, 12 hours, 1 day, 3 days, 5 days, 7 days, 10 days, 14 days, 21 days and 28 days after the start of regeneration and had a total of 345,702,776 strand non-specific singleend reads. Because of the large size of this data set and our goal of testing many different assemblers and parameter settings, we first restricted our analysis to data from three of the time points ( 6 hours, 14 days and 28 days), which made up a total of 55,559,405 reads. We ran Trinity, Oases and SOAPdenovo-Trans on these data to produce over 100 different assemblies, each of which we scored using RSEM-EVAL. Trans-ABySS was not included due to some difficulties in running it.

Since we did not have a known axolotl transcript set, we were unable to use the reference-based measures we have discussed thus far to assess the RSEM-EVAL score's effectiveness for these data. Therefore, to obtain an orthogonal measure of accuracy with which to validate the RSEMEVAL score for this data set, we instead used alignments of the assembly contigs to the known protein sequences of the frog species Xenopus tropicalis. Specifically, we aligned the assemblies against the frog protein sequences with BLASTX [28] and calculated the number of frog proteins that were recovered to various percentages of length by an axolotl contig (Additional file 1: Section 9). We found that, in general, the assemblies with higher RSEMEVAL scores were those that were also considered better by comparison with the Xenopus protein set (Figure 11). Thus, the RSEM-EVAL score appears to be selecting the highest-quality axolotl assemblies.

We then pooled all time points of the time course and built an assembly using the assembler (Trinity) and parameter set (--glue_factor 0.01 --min_iso_ratio 0.1 ) that maximized the RSEMEVAL score on the subset described above. This assembly is publicly available from the DETONATE website. We
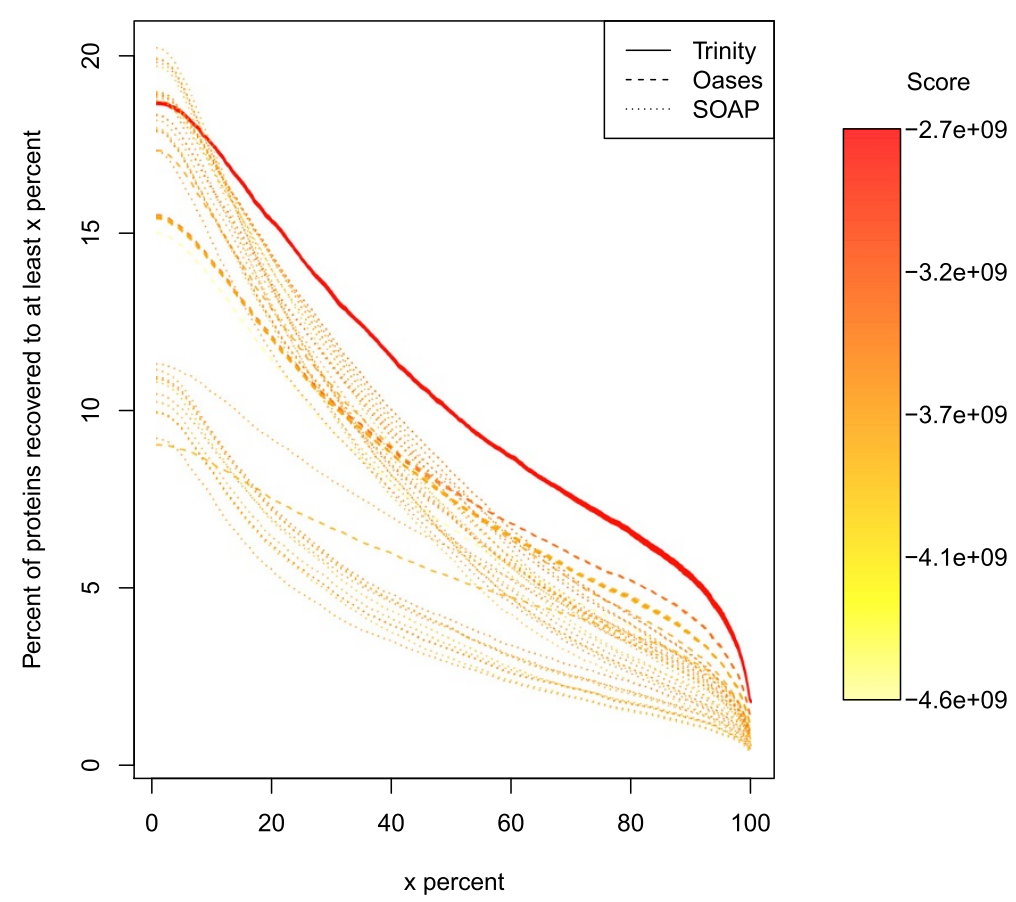

Figure 11 RSEM-EVAL scores and Xenopus protein recovery for the axolotl blastema transcriptome assemblies. The $y$-axis represents the percentage of proteins with at least $x$ percent of their length ( $x$-axis) recovered by an axolotl contig. The curve for each assembly is colored according to its RSEM-EVAL score, with red representing the highest RSEM-EVAL score. The assembly with the curve closest to the upper-right corner is the best in terms of its comparison with the Xenopus protein set. 
compared this assembly to a published axolotl assembly [27]. We find that the new assembly is longer overall and has a larger N50 score than the published assembly (Additional file 1: Table S3).

As length-based measures may not be indicative of a higher quality assembly, we also evaluated the assemblies based on the number of expressed genes and the number of differentially expressed up-regulated (DE UP) genes at each time point in the axolotl RNA-Seq time course. To enable direct comparisons with the published assembly, we used data and methods identical to those in [27], which used a comparative technique that analyzes the axolotl transcripts in terms of their orthologous human genes. With the new RSEM-EVAL guided assembly, we identify both more expressed genes at each time point and more DE UP genes at each time point (Additional file 1: Figure S15). The majority of DE UP genes found in the published assembly are captured in the new assembly (608 of $888=$ $68 \%$ ), while only $39 \%(608$ of 1,576$)$ of the DE UP genes found in the new assembly are captured in the published assembly. The new assembly identifies many new DE UP genes (968) not captured in the old published assembly.

Because transcription factors are important for setting and establishing cell state [29], we further evaluated the list of transcription factors found in the new assembly that are not found in the published assembly across the axolotl RNA-Seq time course. Prior results indicate that oncogenes are up-regulated early in the time course [27]. Using the new assembly we identify two additional DE UP oncogenes (FOSL1 and JUNB) that are not identified using the published assembly [27]. The prior assembly identified many genes associated with limb development and limb regeneration as being DE UP during the middle phase ( 3 to 14 days) of the time course [27]. The new assembly identifies additional limb development and limb regeneration genes during this middle phase such as HOXA11, HOXA13, MSX1, MSX2 and SHOX. HOXA11 and $H O X A 13$ are important specifiers or markers of positional information along the proximal/distal and anterior/posterior axes of the limb [30]. MSX1 and MSX2 have been shown to be expressed in the axolotl blastema [31]. SHOX mutants in humans exhibit short limbs and overall stature [32]. The identification of many more expressed and DE UP genes, a number of which have previous support for involvement in limb regeneration, suggests that the new assembly gives a more comprehensive view of the genes expressed in the axolotl.

\section{Discussion}

\section{Related work}

RSEM-EVAL is related to several recently developed methods for de novo genome and metagenome assembly. Although the evaluation of de novo genome assemblies is a relatively mature area of research [22,33-40], only recently has the need for statistically principled methods been recognized [41]. Notably, Rahman and Pachter [23] developed CGAL, a principled probabilistic modelbased method for evaluating genome assemblies without the ground truth. RSEM-EVAL and CGAL are closely related in that they both make use of the likelihood to score an assembly. However, RSEM-EVAL is necessarily more complex due to important differences between the tasks of transcriptome and genome assembly. In particular, with genome assembly it is generally assumed that all chromosomes are sequenced to the same sequencing depth, whereas with transcriptome assembly one has to consider variable sequencing depth because of widely varying transcript abundances. Therefore, as we demonstrated through our experiments, use of the likelihood alone is suboptimal for evaluating transcriptome assemblies. In fact, even for de novo genome assembly evaluation, one can show that use of the likelihood alone is not optimal. For example, the likelihood criterion cannot distinguish between the true assembly and assemblies constructed by duplicating each contig of the true assembly $c$ times. RSEM-EVAL corrects for this limitation by prior modeling of assemblies.

Because the task of de novo metagenome assembly is roughly equivalent to that of de novo transcriptome assembly, RSEM-EVAL also has close connections to methods developed for metagenomics. Metagenomic data are similar to transcriptomic data in that each distinct element of the population is present at varying multiplicities. In this paper we have shown that RSEM-EVAL has important advantages over the two model-based referencefree measures that have been developed for evaluating metagenome assemblies, Genovo and ALE. In particular, RSEM-EVAL correctly models transcript abundances and read mapping uncertainty, which is critical for distinguishing between assemblies of alternatively spliced transcripts. And on realistic data sets, RSEM-EVAL is notably faster than Genovo and ALE, both of which have runtimes measured in days, which is unlikely to be practical for users interested in evaluating multiple assemblies.

O'Neil and Emrich [20] comprehensively assessed a large number of reference-free and comparativereference-based measures for evaluating de novo transcriptome assemblies and similarly dismissed N50 as a useful measure in this context. They identified a number of informative measures and we compared RSEM-EVAL to three of them. Two of these measures (number of bases in contigs and orthology hit ratio) had poor correlation with the REF-EVAL reference-based measures. The third measure, number of unique proteins matched, performed well in our experiments, further supporting the usefulness of this measure. However, unlike RSEM-EVAL, this is technically a reference-based measure and is dependent on a good comparative reference protein set. In addition, 
this measure is not appropriate as an objective function for a de novo transcriptome assembler because it focuses solely on matching proteins from a closely related species and as such does not take into account how well an assembly explains the reads used in its construction.

\section{Limitations and future work}

We stress that the correlation experiments presented in this study were primarily designed to evaluate the RSEMEVAL score, not to determine which assembler is most accurate, in general. The versions of the assemblers used were not the most recent ones at the time of writing and the parameter variations used were not necessarily those recommended by the assemblers. Nevertheless, with our selected assembler versions and parameter variations (which included the default settings for each assembler), our results provide some evidence that Trinity is more accurate than the other assemblers. To confirm this, future benchmarking will require use of the latest versions of these actively developed assemblers, additional data sets, and more carefully selected parameters.

We also note a couple of important limitations regarding the use of RSEM-EVAL. First, it is critical that RSEMEVAL be run on the same RNA-Seq data set that was provided to the assemblers as it assumes that an assembly is compatible with the data. Second, RSEM-EVAL should not be used if genome information is used during transcriptome assembly because its model is purely based on the RNA-Seq data and therefore might be misleading for genome-guided assemblies. Lastly, RSEM-EVAL currently focuses only on assemblies constructed from Illumina RNA-Seq data. We choose to focus on the Illumina platform as it is currently the most popular RNA-Seq platform. However, the methods we have presented can, in principle, be extended to other sequencing platforms (e.g., Roche 454 sequencing, Ion Torrent and Pacific Biosciences), and we plan to do so in the near future.

In addition to supporting other sequencing platforms, we plan to extend our framework to accommodate indel alignments and sequencing biases. We also plan to investigate remedies to RSEM-EVAL's weakness of scoring assemblies with incorrectly fused contigs above the ground truth. Although it is unlikely that any referencefree measure can score the ground truth above all other assemblies, our random perturbation experiments suggest that RSEM-EVAL is permissive of assemblies that concatenate low-coverage contigs, perhaps because of its modeling of the true assembly with minimum overlap length 0 .

\section{Conclusions}

We presented DETONATE, a methodology and corresponding software package for evaluating de novo transcriptome assemblies, whch can compute both reference-free and reference-based measures. RSEMEVAL, our reference-free measure, uses a novel probabilistic model-based method to compute the joint probability of both an assembly and the RNA-Seq data as an evaluation score. Since it only relies on the RNA-Seq data, it can be used to select a best assembler, tune the parameters of a single assembler and guide new assembler design, even when the ground truth is not available. REF-EVAL, our toolkit for reference-based measures, allows for a more refined evaluation compared to existing reference-based measures. The measures it provides include recall, precision and $F_{1}$ scores at the nucleotide and contig levels, as well as a $\mathrm{KC}$ score.

Experimental results for both simulated and real data sets show that our RSEM-EVAL score accurately reflects assembly quality. Results from perturbation experiments that explored the local assembly space around the ground truth suggest that RSEM-EVAL ranks the ground truth among the locally highest-scoring assemblies. In contrast, a score based only on the likelihood fails to rank the ground truth among its best scores, which highlights the importance of including a prior on assemblies as part of an evaluation score. Through correlation experiments, we measured the similarity of the RSEM-EVAL scores to different reference-based measures. We find that, in general, the RSEM-EVAL score correlates well with referencebased measures. In contrast, simple reference-free measures, such as N50, did not correlate well, suggesting that they are inappropriate for evaluating transcriptome assemblies. And whereas several model-based methods designed for the highly similar task of metagenome assembly evaluation had correlations with reference-based measure comparable to those of RSEM-EVAL, we found that these methods failed in a certain class of realistic scenarios and did not have practical runtimes.

To demonstrate the usage of RSEM-EVAL, we assembled a set of contigs for the regenerating axolotl limb with its guidance. Evaluation of this new assembly suggests that it gives a more comprehensive picture of genes expressed and genes up-regulated during axolotl limb regeneration. Thus, RSEM-EVAL is likely to be a powerful tool in the building of assemblies for a variety of organisms where the genome has not yet been sequenced and/or the transcriptome has not yet been annotated.

\section{Materials and methods}

\section{The true assembly according to DETONATE}

As discussed in the Results above, both RSEM-EVAL and REF-EVAL rely on the concept of the true assembly of a set of RNA-Seq reads, which is the assembly one would construct if given knowledge of the true origin of each read. For each read, $r$, let transcript $(r), \operatorname{left}(r)$ and $\operatorname{right}(r)$, denote the transcript from which $r$ truly originates, the leftmost (5') position of $r$ along the transcript, and the 
rightmost ( $\left.3^{\prime}\right)$ position of $r$ along the transcript, respectively. We parameterize this notion of a true assembly by a length, $w$, which is the minimum overlap between two reads required for the extension of a contig. Given $w$, we define the sequence of a segment, [start, end], of a transcript, $t$, to be a true contig if there exists an ordered set of reads, $\left(r_{1}, r_{2}, \ldots, r_{n}\right)$, such that:

1. $\operatorname{transcript}\left(r_{i}\right)=t, \quad \forall i$

2. $\operatorname{right}\left(r_{i}\right)-\operatorname{left}\left(r_{i+1}\right)+1 \geq w, \quad \forall i<n$

3. $\operatorname{left}\left(r_{1}\right)=$ start

4. $\operatorname{right}\left(r_{n}\right)=$ end

5. [start, end] is maximal

The first four conditions ensure that the segment is completely covered by reads overlapping by at least $w$ bases and the last condition ensures that one contig cannot be contained within another. With single-end data, the true assembly is then defined as the set of all true contigs. Such an assembly is the best theoretically achievable by a de novo assembler that requires at least $w$ bases of overlap to merge two reads. The true assembly at minimum overlap length $w=0$ is the best theoretically achievable assembly in that it represents all contiguously covered segments of the transcript sequences. With paired-end data, one may also consider the notion of a true scaffold, which is a set of true contigs linked together by strings of $N$ characters representing intervals of transcripts that were not covered by any reads but that were spanned by read pairs. More precisely, if one constructs a graph with each true contig as a vertex and an edge between any two vertices whose respective contigs are linked by a read pair, then the set of true scaffolds corresponds to the connected components of this graph.

\section{Overview of RSEM-EVAL}

In this and the following subsections, we describe RSEMEVAL in more detail. To simplify the presentation, we initially restrict our attention to contig assemblies from single-end data. In a later subsection, we will describe the slight differences in RSEM-EVAL when used with scaffold assemblies from paired-end data.

RSEM-EVAL models an RNA-Seq data set, $D$, consisting of $N$ reads, each of length $L$, and an assembly, $A$, consisting of $M$ contigs, with the length of contig $i$ denoted by $\ell_{i}$ and its sequence by $a_{i}$. RSEM-EVAL also models the expected read coverage $\Lambda=\left\{\lambda_{i}\right\}$ of each contig's parent transcript, where the expected read coverage of a transcript is defined as the expected number of reads that start from each valid position of the transcript, given the sequencing throughput, $N$. A transcript's expected read coverage is proportional to its relative expression level (Additional file 1: Section 1).

A natural way to decompose the joint distribution of an assembly and the reads used to construct it, $P(A, D)$, would be to (1) specify a prior distribution over the unobserved transcript sequences and their abundances, (2) model the generation of reads from these transcripts and (3) model $A$ as being the true assembly at minimum overlap length 0 of the reads in $D$ (Additional file 1: Figure S1a). Unfortunately, this decomposition requires us to integrate out the unobserved transcript sequences to obtain the distribution $P(A, D)$, and doing so is computationally infeasible.

Instead, RSEM-EVAL decomposes the joint distribution in an equally valid but more computationally convenient manner, as follows:

1. We specify a prior distribution, $P(A \mid \Lambda)$, over the assembly $A$, given the expected read coverage of each contig's parent transcript, $\Lambda$.

2. We model the generation of a set of reads $D$ consistent with the assembly $A$ and the expected read coverage $\Lambda$; this model defines the likelihood $P(D \mid A, \Lambda)$, the probability of the reads $D$ given the assembly $A$ and the expected read coverage $\Lambda$.

3. Instead of specifying a concrete distribution over the number of contigs $M$ and the expected read coverage $\Lambda$, we approximately integrate out these variables using the BIC [42].

Based on this decomposition, the RSEM-EVAL score, $\log P(A, D)$, can be expressed as a sum of three terms: the assembly prior, the likelihood and a BIC term (Equation 1 in the Results). These three terms are detailed in the next three subsections. The relationship between the natural decomposition and the RSEM-EVAL model is further discussed in Additional file 1: Section 2.

\section{RSEM-EVAL's assembly prior component}

RSEM-EVAL's prior distribution over assemblies is based on a simple parametric model of the transcriptome and the reads, together with the concept of a true assembly as we have described. First, a key assumption of the prior model is that each contig is generated independently. This assumption is useful, even though it is not satisfied in practice since multiple contigs can be generated from the same transcript. With this assumption, we may express the prior as:

$$
P(A \mid \Lambda)=\prod_{i=1}^{M} P\left(a_{i} \mid \lambda_{i}\right)
$$

Second, each contig's parent transcript is modeled as follows.

1. The transcript's length follows a negative binomial distribution, the parameters of which may be estimated from a known transcriptome. 
2. The transcript's sequence follows a uniform distribution, given the transcript's length.

3. For each position in the transcript, the number of reads starting at that position follows a Poisson distribution (mean $=$ expected read coverage), independently, given the transcript length and the expected read coverage.

Third, each contig's distribution is induced from the distribution of its parent transcript, as follows. Imagine that we repeat the following steps until we have a large enough number of contigs in the bag:

1. A transcript and its reads are generated as above.

2. The true assembly at minimum overlap length $w=0$ is constructed from these reads.

3. All contigs in the true assembly are put into the bag. The frequency of contigs in the resulting bag defines our per contig prior $P\left(a_{i} \mid \lambda_{i}\right)$.

The above specification leads to the following functional form for the prior:

$$
P(A \mid \Lambda)=\underbrace{\left(\prod_{i=1}^{M} P\left(\ell_{i} \mid \lambda_{i}\right)\right)}_{\text {contig length prior }} \underbrace{\left(\frac{1}{4}\right)^{\sum_{i=1}^{M} \ell_{i}}}_{\text {assembly size prior }}
$$

One can work out a dynamic programming algorithm to compute $P\left(\ell_{i} \mid \lambda_{i}\right)$, by means of which the prior can be computed efficiently (Additional file 1: Section 3 ).

The practical contribution of the prior is as follows. Each term of the contig length prior, $P\left(\ell_{i} \mid \lambda_{i}\right)$, penalizes contigs with aberrant lengths that are not likely given the expected read coverage of their parent transcripts. The assembly size prior, $\left(\frac{1}{4}\right)^{\sum_{i=1}^{M} \ell_{i}}$, imposes a parsimony preference on the total length of an assembly and pushes the RSEM-EVAL score to favor assemblies using fewer bases to explain the RNA-Seq data.

\section{RSEM-EVAL's likelihood component}

For modeling the RNA-Seq reads, we build on the model used by RSEM [43,44] for the task of transcript quantification. The RSEM model provides a probability distribution for an RNA-Seq data set, $D$, given known transcript sequences, $T$, and relative abundances of those transcripts encoded by the parameters, $\Theta$. Given this model, RSEM uses the expectation-maximization algorithm for efficient computation of the ML estimates for $\Theta$. Unfortunately, we cannot directly use the likelihood under the RSEM model because (1) we do not observe the full-length transcript sequences and (2) we require that the RNA-Seq reads are consistent with an assembly in that they completely cover the contigs. Nevertheless, the RSEM model can be used as part of a generative process that results in a proper probability distribution over $D$, given an assembly, $A$. This process follows a simple two-step rejection procedure:

Step 1: Generate a set of reads, $D^{\prime}$, according to the RSEM model with the contigs in $A$ treated as full-length transcripts.

Step 2: If the reads in $D^{\prime}$ completely cover the contigs, then set $D=D^{\prime}$, otherwise go back to step 1 .

This process results in the following form for RSEMEVAL's likelihood:

$$
P\left(D \mid A, \Lambda_{\mathrm{MLE}}\right)=\frac{P_{\mathrm{RSEM}}\left(D \mid T=A, \Theta_{\mathrm{MLE}}^{c}\right)}{P_{\mathrm{RSEM}}\left(C=1 \mid T=A, \Theta_{\mathrm{MLE}}^{c}\right)},
$$

where $P_{\text {RSEM }}$ denotes a probability under the RSEM model and $C=1$ denotes the event that every position in the assembly is covered by reads that overlap with each other by at least $w$ bases. $\Theta_{\mathrm{MLE}}^{c}$ denotes the equivalent parameter values of the RSEM model given the ML expected read coverage values, $\Lambda_{\mathrm{MLE}}$. We refer to the denominator of Equation 3 as the likelihood correction term. This term can be calculated efficiently (Additional file 1: Section 4).

\section{RSEM-EVAL's BIC penalty component}

The BIC penalty is proportional to the product of the number of free parameters and the logarithm of the size of the data. The free parameters are the expected coverage of each contig, plus one extra parameter for the expected number of reads from RSEM's noise model, for $M+1$ parameters in total. The data size is $N$, which represents the number of reads. The BIC penalty imposes a parsimony preference on the total number of contigs in an assembly.

\section{The inference algorithm used to compute the RSEM-EVAL score}

We use the following heuristic inference algorithm to calculate the RSEM-EVAL score:

1. Learn $\Theta_{\text {MLE }}$ using RSEM, treating the input assembly $A$ as the true transcript set.

2. Convert $\Theta_{\mathrm{MLE}}$ into $\Lambda_{\mathrm{MLE}}$ via the formula:

$$
\lambda_{\mathrm{MLE}, i}=\frac{N \theta_{\mathrm{MLE}, i}}{\ell_{i}+L+1}, \text { for } i>0
$$

3. Calculate the RSEM-EVAL score, $\log P(A, D)$, using $\Lambda_{\mathrm{MLE}}$ and Equation 1.

In step 1, RSEM requires use of a short-read alignment program to generate candidate alignments of each read to the assembly $[43,44]$. The Bowtie aligner (v0.12.9) [45] was used for all experiments except the random perturbation experiments. Bowtie was called through RSEM with RSEM's default Bowtie parameters. For the random perturbation experiments, since the perturbations were 
subtle, we chose to use a more sensitive aligner, GEM (binary pre-release 3) [46]. To produce alignments with similar criteria as Bowtie, we first extracted the first $25 \mathrm{bp}$ (seed) of every read and aligned the seeds using GEM with-q ignore --mismatch-alphabet ACGNT -m 2 -e 0 --max-big-indel-length 0 -s 2 -d 200 -D 0 options set for gem-mapper. Lastly, we filtered any alignments for which the seed could not be extended to the full read length.

In step 2, we do not use the contig-level read coverage, $\frac{N \theta_{\mathrm{MLE}, i}}{\ell_{i}-L+1}$ to approximate the expected read coverage of its parent transcript, $\lambda_{i}$, because it is likely to overestimate $\lambda_{i}$, especially for short contigs. Instead, to make a partial correction for this overestimation, we adjust the denominator in this estimator to $\ell_{i}+L+1$ to account for the fact that no reads started within $L$ bases of the start positions of the reads making up the contig (otherwise, the contig would have been extended, since our model considers the assembly to have been created using minimum overlap length $w=0)$.

\section{RSEM-EVAL with paired-end data}

RSEM-EVAL may also be used to evaluate scaffold assemblies from paired-end data. There are just a few differences between the models RSEM-EVAL uses for single-end and paired-end data. With paired-end data, $N$ becomes the number of read pairs in the data set, $L$ becomes the mean fragment length, and $M$ becomes the number of scaffolds in the assembly. The likelihood component of the RSEMEVAL score uses the probability of the data under RSEM's paired-end model, which includes the probabilities of the fragment lengths implied by the alignments of the read pairs. The likelihood correction term and assembly prior component, both of which are dependent on $L$, are computed based on the mean fragment length rather than the mean read length. The inference algorithm used by RSEM-EVAL for paired-end data is the same as for singleend data with the exception that the reads are aligned to the scaffolds as read pairs rather than as individual reads.

\section{REF-EVAL's estimate of the true assembly}

REF-EVAL estimates a set of true contig (scaffold) sequences from a given set of transcripts and RNA-Seq single-end (paired-end) reads using the following procedure:

1. Align the reads against the transcript sequences using RSEM.

2. For each alignable read (read pair), sample one of its alignments based on the posterior probability that it is the true alignment, as estimated by RSEM. The set of alignments for each read (read pair) includes the null alignment that the read (read pair) comes from background noise.
3. Treat the sampled alignments as true alignments and compute the true contigs with minimum overlap length $w=0$.

4. For paired-end reads, join any true contigs that are spanned by at least one read pair into true scaffolds, with the distance between contigs determined by their positions in the parent transcript.

\section{REF-EVAL's contig- (scaffold-) and nucleotide-level measures}

Given a set of ground truth reference sequences, REFEVAL provides assembly recall, precision and $F_{1}$ scores at two different granularities. Recall is the fraction of reference elements (contigs, scaffolds or nucleotides) that are correctly recovered by an assembly. Precision is the fraction of assembly elements that correctly recover a reference element. The $F_{1}$ score is the harmonic mean of recall and precision:

$$
F_{1}=\frac{2 \times \text { recall } \times \text { precision }}{\text { recall }+ \text { precision }}
$$

Because the $F_{1}$ score is a combination of both recall and precision, it gives a more balanced view of an assembly's accuracy than either precision or recall does alone.

REF-EVAL provides these measures at two different granularities: contig (scaffold) and nucleotide. Taking recall as an example, the measures at the two levels can be summarized as follows. For both levels, we first compute all significant local alignments between the assembly sequences and the reference sequences, using BLAT [47]. The contig (scaffold) level measurement $[2,16,17]$ counts the number of correctly recovered reference sequences after a one-to-one mapping is established between contigs (scaffolds) and reference sequences. At the nucleotide level, the recall measure instead counts the number of correctly recovered nucleotides based on the alignments between the assembly and the ground truth sequences $[5,6]$. The precision measures for both levels are calculated similarly by switching the roles of the assembly sequences and reference sequences.

In detail, REF-EVAL defines the contig (scaffold) recall as follows. For a reference sequence to be correctly recovered, at least $99 \%$ of its (non- $N$ ) sequence must be identical to that of the assembly sequence to which it is aligned, and vice versa, and the total number of insertions and deletions in the alignment between the two must be no more than $1 \%$ of the length of either sequence. Each percentage is computed relative to the length (excluding $N$ characters) of the assembly sequence or the length of the reference sequence, whichever is most stringent. Under these criteria, multiple reference sequences can be recovered by the same assembly sequences. To handle this issue, we define a bipartite graph in which the vertices are the assembly sequences and the reference sequences, and the 
edges correspond to alignments that meet the above criteria. The contig (scaffold) recall is the cardinality of the maximum cardinality matching of this graph.

In detail, REF-EVAL defines the nucleotide recall as follows. A nucleotide in a reference sequence is considered to be correctly recovered if the corresponding nucleotide in the alignment selected for that position is identical. To handle the issue of multiple local alignments overlapping a given reference position, we select a single alignment for each position by picking alignments in order of their marginal contribution to the nucleotide recall, given the alignments that have already been selected. Algorithms to compute the contig and nucleotide measures are given in Additional file 1: Sections 6 and 7.

\section{REF-EVAL's $\boldsymbol{k}$-mer compression score}

The KC score is a combination of two measures, WKR and ICR (Equation 2). WKR measures an assembly's recall of the $k$-mers present in the reference sequences, with each $k$-mer weighted by its relative frequency within the reference transcriptome. It has several advantages over the contig-, scaffold- and nucleotide-level recall measures. First, unlike the nucleotide measure, it takes into account connectivity between nucleotides, but is not as stringent as the contig measure because it only considers connectivity of nucleotides up to $k-1$ positions apart. Second, it is biased toward an assembler's ability to reconstruct transcripts with higher abundance, which are arguably more informative for evaluation because there are generally sufficient data for their assembly. Lastly, it is relatively easy to compute because it does not require an alignment between the assembly and the reference sequences.

To compute WKR, the relative abundances of the reference elements are required. These abundances may be estimated from the RNA-Seq data used for the assembly, and REF-EVAL uses RSEM for this. Given the reference sequences and their abundances, a $k$-mer occurrence frequency profile, $p$, is computed, with individual $k$-mer occurrences weighted by their parent sequences' abundances: for each $k$-mer $r$, we define:

$$
p(r)=\frac{\sum_{b \in B} n(r, b) \tau(b)}{\sum_{b \in B} n(b) \tau(b)}
$$

where $B$ is the set of reference sequences, and for each reference sequence $b$ in $B, n(r, b)$ is the number of times the $k$-mer $r$ occurs in $b, n(b)$ is the total number of $k$-mers in $b$, and $\tau(b)$ is the relative abundance of $b$. Letting $r(A)$ be the set of all $k$-mers in the assembly, WKR is defined as:

$$
\mathrm{WKR}=\sum_{r \in r(A)} p(r)
$$

Since recall measures only tell half of the story regarding accuracy, the KC score includes a second term, ICR, which serves to penalize large assemblies. We define the ICR of an assembly as:

$$
\mathrm{ICR}=\frac{|A|}{N L}
$$

As this term's name suggests, we can view an assembler as a compressor for RNA-Seq data and the assembly it outputs as the compressed result. The uncompressed data size is the total number of nucleotides in the data, $N L$, and the compressed result size is the total number of nucleotides in the assembly, $|A|$. The smaller the ICR value, the more efficient the assembler is in compressing the data. We chose to use ICR over other possible precision-correcting schemes (e.g., $F_{1}$ score) because of important mathematical connections between the RSEMEVAL and KC scores (Additional file 1: Section 8). Thus, as we showed with our experiments, the reference-based $\mathrm{KC}$ score provides some additional intuition for what the reference-free RSEM-EVAL score is measuring. The theoretical links between the two scores suggest that the $\mathrm{KC}$ score would benefit from some additional terms (Additional file 1: Section 8); however, in keeping with our goal of simplicity, we restricted the $\mathrm{KC}$ score to the two most dominant terms.

\section{RNA-Seq data used in the perturbation experiments}

Both the random and the guided perturbation experiments (see Results) use a simulated set of RNA-Seq reads. We simulated these reads from a mouse transcript set (Ensembl Release 63 [48]), using RSEM's [43,44] RNA-Seq simulator and simulation parameters learned from the first mates of a set of real paired-end mouse data (Sequence Read Archive accession [SRA:SRX017794] [49]).

The resulting simulated data set contained around 42 million strand non-specific 76-bp single-end reads. For reasons of computational efficiency, in the random perturbation experiments, we only used reads from transcripts on chromosome 1, and we constructed the ground truth accordingly. This resulted in 1,843,797 reads that formed 10,974 contigs in the ground truth assembly.

For our guided perturbation experiments, we additionally used a real data set consisting of the first mates of the mouse data previously mentioned. This real data set also contained around 42 million strand non-specific 76-bp single-end reads. The true origin of each read in the real data set was estimated using REF-EVAL.

\section{Construction of randomly perturbed assemblies}

The random perturbation experiments (see Results) compare the true assembly's RSEM-EVAL score to the scores of numerous perturbed variants of this assembly. These perturbed assemblies were constructed as follows. Substitution assemblies (randomly perturbed assemblies with 
substitution mutations) were generated by randomly and independently substituting each base of the ground truth at a specific substitution rate. Fusion assemblies were generated by repeatedly joining two randomly selected contigs until the specified number of fusion events was reached. For each join, if the two selected contigs shared a common sequence at their ends to be joined, the contigs were overlapped such that the shared sequence only appeared once in the fused contig. To generate a fission assembly, each contig position at which two reads were merged in the ground truth was independently selected as a fission point at the specified fission rate. If reads overlapped at a fission point, the overlapped segment was made to appear in both contigs after the fission. To generate an indel-perturbed assembly, insertions and deletions were introduced across the ground truth assembly according to the specified indel rate. The length of each indel was sampled from a geometric distribution with mean 3 , to model the short indels occasionally observed in high-throughput sequencing data.

For substitution and indel assemblies, the mutation strength was the mutation rate per contig position, which was varied among $1 \times 10^{-6}, 1 \times 10^{-5}, 1 \times 10^{-4}, 1 \times 10^{-3}$ and $1 \times 10^{-2}$. For example, in the substitution experiment, at mutation rate $1 \times 10^{-3}$, a substitution was introduced at each position of each contig with probability $1 \times 10^{-3}$. The fission experiments used the same range of mutation rates, but with the rate defined as per pair of immediately adjacent and overlapping reads. For fusions, the mutation strength was the number of fusion events, which ranged among 1, 10, 100, 1,000 and 10,000.

\section{RNA sequencing data used in the correlation experiments} For our experiments measuring the correlation between the RSEM-EVAL score and reference-based measures we used five data sets: simulated and real mouse, strand nonspecific single-end RNA-Seq data sets used in the perturbation experiments, the complete strand non-specific paired-end mouse data set [SRA:SRX017794] from which these single-end data sets were derived, and the strandspecific single-end mouse and yeast data sets from Grabherr et al. [4]. The entirety of each data set was used, except for the simulated data set, from which we used roughly half of the simulated reads (reads from transcripts in chromosomes 1 to 10) for computational efficiency. For each assembler, we used a variety of parameter settings (Additional file 2), resulting in a total of 211 assemblies for each of the strand non-specific data sets and 159 assemblies for each of the strand-specific data sets (Table 3). Because the versions of SOAPdenovo-Trans and Trans-ABySS we used did not take into account strand specificity, they were not run on the two strand-specific data sets. The reference-based measures were computed relative to the estimated true assemblies at minimum overlap length $w=0$. These assemblies were estimated using the mouse Ensembl annotation (release 63) for the three mouse data sets and the PomBase [50] Schizosaccharomyces pombe annotation (v09052011) for the yeast data set.

\section{Running of alternative evaluation methods}

The compute_score_denovo program of Genovo (v0.4) was run with default parameters to compute the Genovo score of an assembly from a set of singleend reads. ALE (Git commit b9d2d88915, 29 August 2014), was run with the --metagenome option (to match more closely the transcriptome assembly task) and with the identical alignment file of the reads to the assembly sequences used by RSEM-EVAL. The N50, number of bases in (non-singleton) contigs, number of unique proteins matched and ortholog hit ratio measures were computed using custom scripts. The number of unique proteins matched and ortholog hit ratio measures were computed as previously described [20,26], with one clarification that the ortholog hit ratio of a contig lacking a BLAST hit is defined as zero. For these comparative-reference-based measures, we used the collection of human protein sequences in release 2014_08 of the UniProt Knowledgebase [51] as a reference. For the number of bases in contigs and number of unique proteins matched measures, we removed all assembly elements with length less than or equal to the read length to ensure that only non-singleton contigs were included in the calculations.

\section{Software availability}

The DETONATE software is freely available from its website [52]. Its source-code repository is available [53]. The software runs in POSIX-compatible environments. A copy of the version of the software used for the experiments in this paper is provided as Additional file 3.

Table 3 Assemblers and number of assemblies generated by them (using different parameter settings) for the correlation experiments

\begin{tabular}{llll}
\hline Assembler & Trinity & Oases & SOAPdenovo-Trans \\
\hline Version & r2012-03-17 & 0.2 .06 & 1.01 \\
Number of assemblies & 86 & 73 & 51 \\
\hline
\end{tabular}

SOAPdenovo-Trans and Trans-ABySS were not run on the strand-specific data sets. 


\section{Additional files}

Additional file 1: Supplementary methods, tables and figures.

Additional file 2: Parameter settings for various assemblers.

Additional file 3: Version of the DETONATE source code used for the experiments in this paper.

\section{Abbreviations}

bp, base pair; DE UP, differentially expressed up-regulated; ICR, inverse compression rate; $\mathrm{KC}$ score, $k$-mer compression score; $\mathrm{ML}$, maximum likelihood; RNA-Seq, RNA sequencing; WKR, weighted $k$-mer recall.

\section{Competing interests}

The authors declare that they have no competing interests.

\section{Authors' contributions}

CND conceived the research. BL, NF and CND designed RSEM-EVAL and REF-EVAL. BL implemented RSEM-EVAL. NF implemented REF-EVAL and assembled the DETONATE package. BL and NF performed the analysis. YB, $M C$, JAT and RS contributed the axolotl data and performed axolotl-related analysis. BL, NF, RS and CND wrote the manuscript. All authors read and approved the final manuscript.

\section{Acknowledgements}

We thank Lior Pachter, Sreeram Kannan and Marcel H Schulz for helpful discussions and three anonymous reviewers for constructive comments. This work was supported by the National Institutes of Health (grants R01HG005232 and T15LM007359).

\section{Author details}

${ }^{1}$ Center for RNA Systems Biology, University of California, Berkeley, CA 94720, USA. ${ }^{2}$ Department of Computer Sciences, University of Wisconsin, Madison, WI 53706, USA. ${ }^{3}$ Department of Biology, Indiana State University, Terre Haute, IN 47809, USA. ${ }^{4}$ Morgridge Institute for Research, Madison, WI 53707, USA. ${ }^{5}$ Department of Molecular, Cellular and Developmental Biology, University of California, Santa Barbara, CA 93106, USA. ${ }^{6}$ Department of Cell and Regenerative Biology, University of Wisconsin, Madison, WI 53706, USA. ${ }^{7}$ Department of Biostatistics and Medical Informatics, University of Wisconsin, Madison, WI 53706, USA.

Received: 6 July 2014 Accepted: 30 October 2014

Published online: 21 December 2014

\section{References}

1. Martin J, Bruno VM, Fang Z, Meng X, Blow M, Zhang T, Sherlock G, Snyder M, Wang Z: Rnnotator: an automated de novo transcriptome assembly pipeline from stranded RNA-Seq reads. BMC Genomics 2010, 11:663.

2. Robertson G, Schein J, Chiu R, Corbett R, Field M, Jackman SD, Mungall K, Lee S, Okada HM, Qian JQ, Griffith M, Raymond A, Thiessen N, Cezard T, Butterfield YS, Newsome R, Chan SK, She R, Varhol R, Kamoh B, Prabhu A-L, Tam A, Zhao Y, Moore RA, Hirst M, Marra MA, Jones SJ, Hoodless PA, Birol I: De novo assembly and analysis of RNA-seq data. Nat Methods 2010, 7:909-912.

3. Surget-Groba Y, Montoya-Burgos Jl: Optimization of de novo transcriptome assembly from next-generation sequencing data. Genome Res 2010, 20:1432-1440.

4. Grabherr MG, Haas BJ, Yassour M, Levin JZ, Thompson DA, Amit I, Adiconis X, Fan L, Raychowdhury R, Zeng Q, Chen Z, Mauceli E, Hacohen N, Gnirke A, Rhind N, di Palma F, Birren BW, Nusbaum C, Lindblad-Toh K, Friedman N, Regev A: Full-length transcriptome assembly from RNA-Seq data without a reference genome. Nat Biotechnol 2011, 29:644-652.

5. Chen C-C, Lin W-D, Chang Y-J, Chen C-L, Ho J-M: Enhancing de novo transcriptome assembly by incorporating multiple overlap sizes. ISRN Bioinformatics 2012, 2012:816402.
6. Schulz MH, Zerbino DR, Vingron M, Birney E: Oases: robust de novo RNA-seq assembly across the dynamic range of expression levels. Bioinformatics 2012, 28:1086-1092.

7. Chu H-T, Hsiao WWL, Chen J-C, Yeh T-J, Tsai M-H, Lin H, Liu Y-W, Lee S-A, Chen C-C, Tsao TTH, Kao C-Y: EBARDenovo: highly accurate de novo assembly of RNA-Seq with efficient chimera-detection. Bioinformatics 2013, 29:1004-1010.

8. Xie Y, Wu G, Tang J, Luo R, Patterson J, Liu S, Huang W, He G, Gu S, Li S, Zhou X, Lam T-W, Li Y, Xu X, Wong GK, Wang J: SOAPdenovo-Trans de novo transcriptome assembly with short RNA-Seq reads. Bioinformatics 2014, 30:1660-1666.

9. Huang X, Madan A: CAP3: a DNA sequence assembly program. Genome Res 1999, 9:868-877.

10. Chevreux B, Pfisterer T, Drescher B, Driesel AJ, Müller WE, Wetter T, Suhai $S$ : Using the miraEST assembler for reliable and automated mRNA transcript assembly and SNP detection in sequenced ESTs. Genome Res 2004, 14:1147-1159.

11. Margulies M, Egholm M, Altman WE, Attiya S, Bader JS, Bemben LA, Berka J, Braverman MS, Chen YJ, Chen Z, Dewell SB, Du L, Fierro JM, Gomes XV, Godwin BC, He W, Helgesen S, Ho CH, Irzyk GP, Jando SC, Alenquer ML, Jarvie TP, Jirage KB, Kim JB, Knight JR, Lanza JR, Leamon JH, Lefkowitz SM, Lei M, Li J, et al: Genome sequencing in microfabricated high-density picolitre reactors. Nature 2005, 437:376-380.

12. Zheng Y, Zhao L, Gao J, Fei Z: iAssembler: a package for de novo assembly of Roche-454/Sanger transcriptome sequences. $B M C$ Bioinformatics 2011, 12:453.

13. Kumar S, Blaxter ML: Comparing de novo assemblers for 454 transcriptome data. BMC Genomics 2010, 11:571.

14. Feldmeyer B, Wheat CW, Krezdorn N, Rotter B, Pfenninger M: Short read Illumina data for the de novo assembly of a non-model snail species transcriptome (Radix balthica, Basommatophora, Pulmonata), and a comparison of assembler performance. $B M C$ Genomics 2011, 12:317.

15. Zhao $Q-Y$, Wang $Y$, Kong $Y-M, L$, Lo $D, L i X$, Hao P: Optimizing de novo transcriptome assembly from short-read RNA-Seq data: a comparative study. BMC Bioinformatics 2011, 12:S2.

16. Mundry M, Bornberg-Bauer E, Sammeth M, Feulner PGD: Evaluating characteristics of de novo assembly software on 454 transcriptome data: a simulation approach. PLoS One 2012, 7:e31410.

17. Ren $X$, Liu T, Dong J, Sun L, Yang J, Zhu Y, Jin Q: Evaluating de Bruijn graph assemblers on $\mathbf{4 5 4}$ transcriptomic data. PLOS One 2012, 7:e51188.

18. Clarke K, Yang Y, Marsh R, Xie L, Zhang KK: Comparative analysis of de novo transcriptome assembly. Sci China Life Sci 2013, 56:156-162.

19. Lu B, Zeng Z, Shi T: Comparative study of de novo assembly and genome-guided assembly strategies for transcriptome reconstruction based on RNA-Seq. Sci China Life Sci 2013, 56:143-155.

20. O'Neil ST, Emrich SJ: Assessing de novo transcriptome assembly metrics for consistency and utility. BMC Genomics 2013, 14:465.

21. Miller JR, Koren S, Sutton G: Assembly algorithms for next-generation sequencing data. Genomics 2010, 95:315-327.

22. Salzberg SL, Phillippy AM, Zimin A, Puiu D, Magoc T, Koren S, Treangen TJ, Schatz MC, Delcher AL, Roberts M, Marçais G, Pop M, Yorke JA: GAGE: a critical evaluation of genome assemblies and assembly algorithms. Genome Res 2012, 22:557-567.

23. Rahman $A$, Pachter L: CGAL: computing genome assembly likelihoods. Genome Biol 2013, 14:R8.

24. Laserson J, Jojic V, Koller D: Genovo de novo assembly for metagenomes. J Comput Biol 2011, 18:429-443.

25. Clark SC, Egan R, Frazier PI, Wang Z: ALE: a generic assembly likelihood evaluation framework for assessing the accuracy of genome and metagenome assemblies. Bioinformatics 2013, 29:435-443.

26. O'Neil ST, Dzurisin JDK, Carmichael RD, Lobo NF, Emrich SJ, Hellmann JJ: Population-level transcriptome sequencing of nonmodel organisms Erynnis propertius and Papilio zelicaon. BMC Genomics 2010, 11:310.

27. Stewart R, Rascon CA, Tian S, Nie J, Barry C, Chu LF, Ardalani H, Wagner RJ, Probasco MD, Bolin JM, Leng N, Sengupta S, Volkmer M, Habermann B, Tanaka EM, Thomson JA, Dewey CN: Comparative RNA-seq analysis in 
the unsequenced axolotl: the oncogene burst highlights early gene expression in the blastema. PLoS Comput Biol 2013, 9:e1002936.

28. Altschul SF, Madden TL, Schäffer AA, Zhang J, Zhang Z, Miller W, Lipman DJ: Gapped BLAST and PSI-BLAST: a new generation of protein database search programs. Nucleic Acids Res 1997, 25:3389-3402.

29. Tam W-L, Lim B: Genome-wide transcription factor localization and function in stem cells. In StemBook. Edited by Girard L. Cambridge, MA Harvard Stem Cell Institute; 2008

30. Zakany J, Duboule D: The role of $j$ Hox genes during vertebrate limb development. Curr Opin Genet Dev 2007, 17:359-366.

31. Koshiba K, Kuroiwa A, Yamamoto H, Tamura K, Ide H: Expression of Msx genes in regenerating and developing limbs of axolotl. J Exp Zool 1998, 282:703-714.

32. Clement-Jones M, Schiller S, Rao E, Blaschke RJ, Zuniga A, Zeller R, Robson SC, Binder G, Glass I, Strachan T, Lindsay S, Rappold GA: The short stature homeobox gene SHOX is involved in skeletal abnormalities in Turner syndrome. Hum Mol Genet 2000, 9:695-702.

33. Phillippy AM, Schatz MC, Pop M: Genome assembly forensics: finding the elusive mis-assembly. Genome Biol 2008, 9:R55.

34. Darling AE, Tritt A, Eisen JA, Facciotti MT: Mauve assembly metrics. Bioinformatics 2011, 27:2756-2757.

35. Earl D, Bradnam K, St John J, Darling A, Lin D, Fass J, Yu HOK, Buffalo V, Zerbino DR, Diekhans M, Nguyen N, Ariyaratne PN, Sung W-K, Ning Z, Haimel M, Simpson JT, Fonseca NA, Birol I, Docking TR, Ho IY, Rokhsar DS, Chikhi R, Lavenier D, Chapuis G, Naquin D, Maillet N, Schatz MC, Kelley DR, Phillippy AM, Koren S, et al: Assemblathon 1: a competitive assessment of de novo short read assembly methods. Genome Res 2011, 21:2224-2241.

36. Lin Y, Li J, Shen H, Zhang L, Papasian CJ, Deng H-W: Comparative studies of de novo assembly tools for next-generation sequencing technologies. Bioinformatics 2011, 27:2031-2037.

37. Narzisi G, Mishra B: Comparing de novo genome assembly: the long and short of it. PLOS One 2011, 6:e19175.

38. Zhang W, Chen J, Yang Y, Tang Y, Shang J, Shen B: A practical comparison of de novo genome assembly software tools for next-generation sequencing technologies. PLoS One 2011, 6:e17915

39. Vezzi F, Narzisi G, Mishra B: Feature-by-feature - evaluating de novo sequence assembly. PLoS One 2012, 7:e31002.

40. Gurevich A, Saveliev V, Vyahhi N, Tesler G: QUAST: quality assessment tool for genome assemblies. Bioinformatics 2013, 29:1072-1075.

41. Howison M, Zapata F, Dunn CW: Toward a statistically explicit understanding of de novo sequence assembly. Bioinformatics 2013, 29:2959-2963.

42. Schwarz G: Estimating the dimension of a model. Ann Stat 1978, 6:461-464.

43. Li B, Ruotti V, Stewart RM, Thomson JA, Dewey CN: RNA-Seq gene expression estimation with read mapping uncertainty. Bioinformatics 2010, 26:493-500.

44. Li B, Dewey CN: RSEM: accurate transcript quantification from RNA-Seq data with or without a reference genome. $B M C$ Bioinformatics 2011, 12:323.

45. Langmead B, Trapnell C, Pop M, Salzberg SL: Ultrafast and memory-efficient alignment of short DNA sequences to the human genome. Genome Biol 2009, 10:R25.

46. Marco-Sola S, Sammeth M, Guigó R, Ribeca P: The GEM mapper: fast, accurate and versatile alignment by filtration. Nat Methods 2012, 9:1185-1188.

47. Kent WJ: BLAT - the BLAST-like alignment tool. Genome Res 2002 , 12:656-664.

48. Flicek P, Amode MR, Barrell D, Beal K, Brent S, Chen Y, Clapham P, Coates G, Fairley S, Fitzgerald S, Gordon L, Hendrix M, Hourlier T, Johnson N, Kähäri A, Keefe D, Keenan S, Kinsella R, Kokocinski F, Kulesha E, Larsson P, Longden I, McLaren W, Overduin B, Pritchard B, Riat HS, Rios D, Ritchie GRS, Ruffier M, Schuster M, et al: Ensembl 2011. Nucleic Acids Res 2011, 39:D800-D806.

49. Trapnell C, Williams B, Pertea G, Mortazavi A, Kwan G, van Baren M, Salzberg SL, Wold B, Pachter L: Transcript assembly and quantification by RNA-Seq reveals unannotated transcripts and isoform switching during cell differentiation. Nat Biotechnol 2010, 28:511-515.

50. Wood V, Harris MA, McDowall MD, Rutherford K, Vaughan BW, Staines DM, Aslett M, Lock A, Bähler J, Kersey PJ, Oliver SG: PomBase: a comprehensive online resource for fission yeast. Nucleic Acids Res 2012, 40:D695-D699.

51. Magrane $\mathrm{M}$, UniProt Consortium: UniProt knowledgebase: a hub of integrated protein data. Database 2011, 2011:bar009.

52. DETONATE website [http://deweylab.biostat.wisc.edu/detonate]

53. DETONATE source code [https://github.com/deweylab/detonate]

\section{Submit your next manuscript to BioMed Central and take full advantage of:}

- Convenient online submission

- Thorough peer review

- No space constraints or color figure charges

- Immediate publication on acceptance

- Inclusion in PubMed, CAS, Scopus and Google Scholar

- Research which is freely available for redistribution 